High-frequency self-excited oscillations in a collapsible-channel flow

Jensen, Oliver E. and Heil, Matthias

2003

MIMS EPrint: 2006.238

Manchester Institute for Mathematical Sciences

School of Mathematics

The University of Manchester

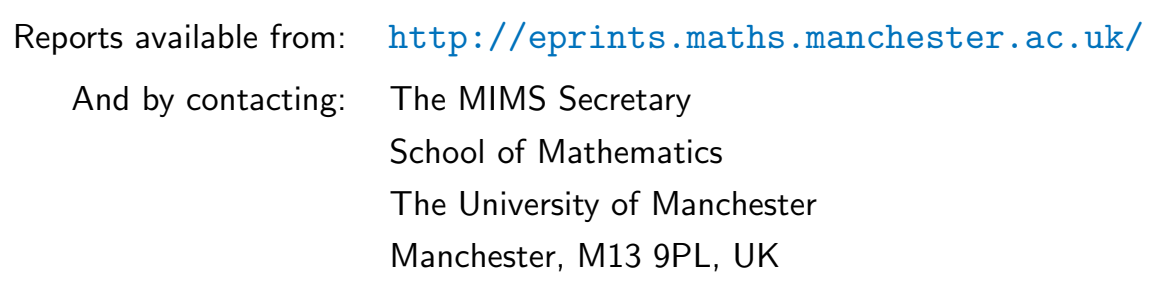

ISSN 1749-9097 


\title{
High-frequency self-excited oscillations in a collapsible-channel flow
}

\author{
By OLIVER E. JENSEN ${ }^{1}$ AND MATTHIAS HEIL ${ }^{2}$ \\ ${ }^{1}$ Division of Applied Mathematics, School of Mathematical Sciences, University of Nottingham, \\ University Park, Nottingham, NG7 2RD, UK \\ ${ }^{2}$ Department of Mathematics, University of Manchester, Oxford Road, Manchester M13 9PL, UK
}

(Received 15 May 2002 and in revised form 9 October 2002)

High-Reynolds-number asymptotics and numerical simulations are used to describe two-dimensional, unsteady, pressure-driven flow in a finite-length channel, one wall of which contains a section of membrane under longitudinal tension. Asymptotic predictions of stability boundaries for small-amplitude, high-frequency, self-excited oscillations are derived in the limit of large membrane tension. The oscillations are closely related to normal modes of the system, which have a frequency set by a balance between membrane tension and the inertia of the fluid in the entire channel. Oscillations can grow by extracting kinetic energy from the mean Poiseuille flow faster than it is lost to viscous dissipation. Direct numerical simulations, based on a fully coupled finite-element discretization of the equations of large-displacement elasticity and the Navier-Stokes equations, support the predicted stability boundaries, and are used to explore larger-amplitude oscillations at lower tensions. These are characterized by vigorous axial sloshing motions superimposed on the mean flow, with transient secondary instabilities being generated both upstream and downstream of the collapsible segment.

\section{Introduction}

Most internal flows in the body pass through deformable vessels. In some instances, flow-induced forces are large enough to cause significant vessel deformation, and the resulting flow-structure interactions can then lead to physiologically significant phenomena. These include flow limitation (whereby airway deformation limits the rate at which air can be forcibly expelled from the lungs, for example) and noise-generating instabilities (such as wheezing in lung airways and Korotkoff sound generation in the compressed brachial artery during sphygmomanometry). A popular and simple device for investigating these phenomena is the Starling Resistor, in which a high-Reynoldsnumber flow is driven through a thin-walled elastic tube that is mounted between two rigid tubes and is subject to external compression. When the elastic tube is compressed sufficiently to buckle along part of its length, thereby becoming highly compliant, the system readily exhibits vigorous flow-induced oscillations. These oscillations arise in multiple distinct modes, and demonstrate many hallmarks of a complex nonlinear dynamical system (Bertram, Raymond \& Pedley 1990, 1991). Understanding the origins and mechanisms of these oscillations remains a significant challenge, since the problem involves a large-amplitude, unsteady, three-dimensional, high-Reynoldsnumber, flow-structure interaction. 


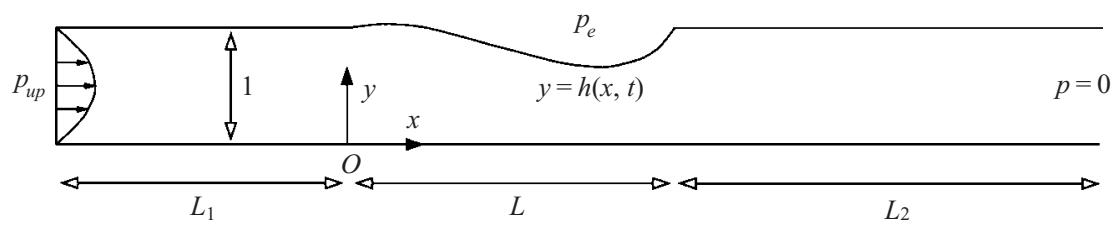

FIGURE 1. The flow domain, showing dimensionless variables and parameters. The membrane occupies $0<x<L$; the channel is rigid otherwise.

The intriguing behaviour of the Starling Resistor has motivated a large number of investigations using simplified theoretical models, which are reviewed in detail in Shapiro (1977), Kamm \& Pedley (1989), Pedley \& Luo (1998) and Heil \& Jensen (2003). Very briefly, these originated with lumped-parameter (ODE-based) approaches that are able to reproduce oscillatory behaviour (e.g. Bertram \& Pedley 1982), and progressed to spatially one-dimensional (but necessarily ad hoc) models that capture potentially important aspects of the flow such as wave propagation and dissipation due to internal flow separation (e.g. Cancelli \& Pedley 1985; Jensen 1990, 1992). Both classes of model show that, to reproduce self-excited oscillations successfully, it is necessary to have some representation of viscous dissipation in the system, and that some account must also be taken of the dissipation and fluid inertia in the rigid parts of the apparatus. Substantial recent progress towards simulating the Starling Resistor has come from studies of a simpler two-dimensional system, in which a flow is driven through a finite-length planar channel, one wall of which contains a (typically massless) membrane under tension that is subject to external compression (see figure 1). This two-dimensional system is the subject of the present paper, and is discussed in detail below. Significant advances have also been achieved computationally for steady, three-dimensional flows in elastic tubes (Heil 1997, 1998; Hazel \& Heil 2003). Also relevant are theoretical studies of small-amplitude, flowinduced instabilities in infinitely long compliant channels (e.g. Grotberg \& Reiss 1984; Gajjar \& Sibanda 1996; Davies \& Carpenter 1997), which reveal potential intrinsic modes of instability (Tollmien-Schlichting waves, travelling-wave flutter, static divergence and their interactions) that are undoubtedly present in a finitelength system but are not so easily characterized in this case.

Computations of steady and unsteady two-dimensional flow in the deformable channel configuration illustrated in figure 1 were presented in a series of papers by Rast (1994) and Luo \& Pedley (1995, 1996, 1998, 2000). Working in a limited region of parameter space, Luo \& Pedley showed how this system exhibits self-excited oscillations provided the Reynolds number of the flow is sufficiently high and the membrane tension sufficiently low. In the stationary state, the membrane can be indented under negative transmural (internal minus external) pressure, and the flow typically separates beyond the point of strongest collapse near the downstream end of the membrane. When the membrane oscillates, the largely inviscid flow in the core of the channel develops a propagating wavy structure downstream of the constriction, very similar to the 'vorticity waves' observed experimentally and computationally in channel flows that are forced either by unsteady localized wall indentations (Pedley \& Stephanoff 1985; Ralph \& Pedley 1988, 1989) or by unsteady pressure gradients in channels with fixed asymmetric indentations (Sobey 1985; Tutty 1992; Tutty \& Pedley 1993; Rosenfeld 1995). Whether the vorticity waves are a cause or an effect of the primary self-excited oscillations in Luo \& Pedley's computations remains an open question. 
The spatial non-uniformity and presence of internal separation in the two-dimensional deformable-channel problem provide significant challenges to any analytical description of this system. However, interactive boundary-layer theory for highReynolds-number flow, building on the framework laid down by Smith $(1976 a, b)$ in which viscous boundary layers on each wall of a weakly indented asymmetric channel are coupled by transverse displacements of the inviscid core flow, has yielded some significant insights. A steady version of Smith's theory was used by Guneratne (1999) to compute possible static membrane configurations in the system shown in figure 1: she showed that a family of static, spatially non-uniform eigenmodes bifurcates from the axially uniform state as the membrane tension is reduced. Under external compression, which acts as a symmetry-breaking perturbation, the membrane can assume either one or many or no axially non-uniform configurations, depending on the parameter values, although the stability of these states was not analysed. Previously, an unsteady version of Smith's theory had been used by Bogdanova \& Ryzhov (1983) to show how downstream-propagating lower-branch Tollmien-Schlichting waves in a rigid-walled channel can grow spatially if $S t R e^{3 / 7}$ exceeds a critical value. Here $R e \equiv \rho U_{0} a / \mu \gg 1$ is the Reynolds number (defined with respect to fluid density $\rho$, viscosity $\mu$, mean flow speed $U_{0}$ and channel width $a$ ) and $S t \equiv \omega a / U_{0} \ll 1$ is the Strouhal number (based on wave frequency $\omega$ ). For $S t R e^{3 / 7} \gg 1$, the waves grow into what can be identified as vorticity waves; they are sustained through an inviscid mechanism and have a wavelength $O\left(S t^{-1 / 3}\right) \gg 1$ that is approximately independent of $R e$; in this limit, the waves can be described by a linearized $\mathrm{KdV}$ equation (e.g. Pedley \& Stephanoff 1985) relating core streamline displacement to wall displacement. At present, however, there is no rational analytical description of the system in figure 1 that shows how an unsteady flow-structure interaction leads to the generation of self-excited oscillations.

Relatively little attention has been paid to high-frequency $(S t \gg 1)$ oscillatory flows in non-uniform channels. Of those studies that are relevant to this paper, Secomb (1979) examined high-frequency motions in a channel with rapidly fluctuating indentations in which unsteadiness is significant both in the core and in viscous boundary layers (showing for example that channel asymmetry has a weak effect, in contrast to the low-St limit); Duck (1979) treated the case of steady, fully developed flow interacting with small-amplitude unsteady symmetric wall motion, including the case in which the flow becomes unsteady in the core, and showed how steady streaming is generated in oscillatory Stokes layers; and Rosenfeld (1995) showed numerically how vorticity waves are suppressed at large $S t$ during oscillatory flow in a rigid indented channel.

In this paper we revisit the two-dimensional deformable channel problem shown in figure 1, treating it with combined asymptotic and computational approaches. Our primary aim is to establish a clear physical mechanism for the generation of self-excited oscillations. In order to develop a rational asymptotic approximation, we consider the limit of large membrane tension, high $R e$ and long axial lengthscales. An advantage of taking tension to be large (and the external pressure sufficiently small) is that membrane deflections are generally small and disturbances are of high frequency $(S t \gg 1)$, so that internal flow separation is suppressed and vorticity perturbations are confined to thin oscillatory Stokes layers at the channel walls. Taking long axial lengthscales allows us to use boundary-layer approximations throughout. We find (in $\S 3$ ) that, for $R e \gg 1$, the system exhibits inviscid normal modes: these are high-frequency oscillations of the membrane that drive axial sloshing motions of the internal flow, with the inertia of the fluid in the entire channel 
balancing elastic restoring forces in the membrane. If these oscillations can extract kinetic energy from the mean flow faster than it is lost to viscous dissipation, the oscillations can grow to become 'self-excited.' We find that steady streaming induced by Reynolds stresses acting in the Stokes layers can be involved in the transfer of energy between the mean flow and the oscillation, and we finally derive an explicit stability threshold for the onset of self-excited oscillations (see (3.48b), (3.51) below). Navier-Stokes simulations $(\S 4)$, based on a fully coupled finite-element discretization of the equations of large-displacement elasticity and the Navier-Stokes equations, are then used to verify the accuracy of the asymptotic predictions for small-amplitude oscillations. The computations are extended to larger amplitudes and lower membrane tensions to demonstrate that the primary mechanism of instability is preserved in this regime, although the flow is complicated substantially by the generation of secondary instabilities.

\section{The model}

We consider a planar channel of total length $L_{0}$, one wall of which contains a segment of thin elastic membrane under longitudinal tension and subject to an external pressure $p_{\text {ext }}$. The channel walls elsewhere are rigid, and the membrane is assumed massless and free of any internal dissipation. When the membrane is flat, the channel has uniform width $a$. Fluid of viscosity $\mu$ and constant density $\rho$ is driven through the channel either by an imposed pressure drop $p_{0}$ or by a prescribed volume flux $q_{0}$.

\subsection{The fluid equations}

Given $p_{0}$, we introduce a velocity scale $U_{0}=p_{0} a^{2} /\left(12 \mu L_{0}\right)$; alternatively, given $q_{0}$ we introduce a velocity scale $U_{0}=q_{0} / a$. We scale all lengths on $a$, time on $a / U_{0}$ and pressures on $\rho U_{0}^{2}$. We introduce Cartesian coordinates $(x, y)$ with origin $O$ (see figure 1) so that the channel walls lie at $y=0$ and $y=1$ (where the channel walls are rigid, that is for $-L_{1} \leqslant x<0$ and $\left.L<x \leqslant L+L_{2}\right)$, or $y=0$ and $y=h(x, t)$ (where the channel is deformable, for $0 \leqslant x \leqslant L$ ). Here $h(0, t)=h(L, t)=1$ for all time $t$, and $a\left(L_{1}+L+L_{2}\right)=L_{0}$.

The flow in the channel is governed by the dimensionless Navier-Stokes equations

$$
\nabla \cdot \boldsymbol{u}=0, \quad \frac{\mathrm{D} \boldsymbol{u}}{\mathrm{D} t}=-\nabla p+\frac{1}{R e} \nabla^{2} \boldsymbol{u},
$$

where $R e=\rho a U_{0} / \mu$, subject to no-slip and no-penetration conditions on the rigid channel walls $\left(\boldsymbol{u}=\mathbf{0}\right.$ on $y=0,-L_{1} \leqslant x \leqslant L+L_{2}$ and $y=1,-L_{1} \leqslant x \leqslant 0, L \leqslant$ $\left.x \leqslant L+L_{2}\right)$. At either end of the channel we impose

$$
\begin{array}{lll}
p=p_{u p} \equiv 12\left(L_{1}+L_{2}+L\right) / \text { Re } \quad \text { or } \quad q=\int_{0}^{1} \boldsymbol{u} \mathrm{d} y=1 & \left(x=-L_{1}\right), \\
p=0 \quad \text { or } \quad q=1 & & \left(x=L+L_{2}\right) .
\end{array}
$$

\subsection{The wall equations}

To describe the wall deformation, we parameterize the position vector to material points on the undeformed wall by the Lagrangian coordinate $\zeta \in[0, L]$ such that $\boldsymbol{r}_{w}(\zeta)=(\zeta, 1)^{T}$. The displacement field $\boldsymbol{d}=\left(d^{(x)}, d^{(y)}\right)^{T}$ displaces material points to their new position $\boldsymbol{R}_{w}(\zeta, t)=\boldsymbol{r}_{w}(\zeta)+\boldsymbol{d}(\zeta, t)$. In the undeformed position, the elastic wall is subject to a large initial stress $\sigma_{0}^{*}=T_{0} / h_{0}$ (where $h_{0}$ is the wall thickness and $T_{0}$ the initial longitudinal tension) which we assume to be much larger than 
the additional stress generated by the wall deformation. This allows us to assume incrementally linear elastic behaviour which implies that $\sigma=\sigma_{0}+\gamma$, where $\sigma=\sigma^{*} / E$ is the dimensionless second Piola-Kirchhoff stress and $\sigma_{0}=\sigma_{0}^{*} / E$ the dimensionless pre-stress. $E$ is the incremental Young's modulus and $\gamma$ is the geometrically nonlinear extensional strain, $\gamma=d_{\zeta}^{(x)}+\frac{1}{2}\left(\left(d_{\zeta}^{(x)}\right)^{2}+\left(d_{\zeta}^{(y)}\right)^{2}\right)$, where a subscript denotes a partial derivative. The wall deformation is governed by the principle of virtual displacements

$$
\int_{0}^{L}\left[\left(\sigma_{0}+\gamma\right) \delta \gamma+\frac{1}{12}\left(\frac{h_{0}}{a}\right)^{2} \kappa \delta \kappa-\left(\frac{a}{h_{0}}\right) \boldsymbol{f} \cdot \delta \boldsymbol{R}_{w} \Delta\right] \mathrm{d} \zeta=0,
$$

where $f=f^{*} / E$ is the non-dimensional traction acting on the wall, with

$$
\Delta \equiv \sqrt{\left(1+d_{\zeta}^{(x)}\right)^{2}+\left(d_{\zeta}^{(y)}\right)^{2}} \quad \text { and } \quad \kappa=\left(d_{\zeta \zeta}^{(y)}\left(1+d_{\zeta}^{(x)}\right)-d_{\zeta \zeta}^{(x)} d_{\zeta}^{(y)}\right) / \Delta,
$$

where $\kappa$ is the wall curvature. The first two terms in (2.3) represent the variation in the wall strain energy due to its extension and bending, respectively. The last term represents the virtual work done by the fluid traction $f$ acting on the deformed wall.

Fluid and solid interact via the no-slip and kinematic condition

$$
\boldsymbol{u}=\frac{\partial \boldsymbol{R}_{w}}{\partial t} \quad \text { on the wall, }
$$

and via the traction

$$
\boldsymbol{f}=\frac{\rho U_{0}^{2}}{E}\left(\left(-p_{e}+p\right) \boldsymbol{I}-\frac{1}{R e}\left(\nabla \boldsymbol{u}+\nabla \boldsymbol{u}^{T}\right)\right) \cdot \boldsymbol{N},
$$

where $N=\left(-d_{\zeta}^{(y)}, 1+d_{\zeta}^{(x)}\right)^{T} / \Delta$ is the outer normal on the wall.

Equation (2.6) shows that at high $R e$, the load on the wall is dominated by the normal traction $\left(-p_{e}+p\right) N$. For wall deformations of small amplitude and long wavelength, material points on the wall will therefore move predominantly in the vertical direction so that $\zeta \approx x$ and $h \approx 1+d^{(x)}$. Furthermore, bending effects can be neglected. In this case equations (2.5) and (2.6) can be formulated as approximate kinematic and normal-stress conditions for the fluid, such that on $y=h, 0 \leqslant$ $x \leqslant L$

$$
u=0, \quad v=h_{t}, \quad p=p_{e}-T h_{x x}\left(1+h_{x}^{2}\right)^{-3 / 2},
$$

where $T=T_{0} /\left(\rho U_{0}^{2} a\right)$. The viscous contribution to the normal stress has been discarded from $(2.7 c)$, a step that is justified in more detail below.

The boundary conditions (2.7) are used for the asymptotic analysis below while (2.5) and (2.6) are used for the numerical simulations. To facilitate comparisons with the analytical results, all computations were performed with a small wall thickness of $h_{0} / a=10^{-2}$ and with 'pinned' boundary conditions (applied by enforcing only $\boldsymbol{d}=\mathbf{0}$ at $\zeta=0, L)$. This ensured that bending effects remained negligible throughout the domain. Furthermore, the axial pre-stress was set to a large value of $\sigma_{0}=10^{3}$. This ensured that, even for the largest wall deformations considered in this study, the tension $\sigma=\sigma_{0}+\gamma$ changed by less than $2 \times 10^{-3} \%$. Thus with fixed values of $\sigma_{0} \gg 1$ and $h_{0} / a \ll 1$, the problem reduces to one with six dimensionless parameters:

$$
\epsilon \equiv \frac{1}{L}, \quad L_{1}, \quad L_{2}, \quad R e=\frac{\rho a U_{0}}{\mu}, \quad T=\frac{T_{0}}{\rho U_{0}^{2} a}, \quad p_{e}=\frac{p_{\text {ext }}}{\rho U_{0}^{2}} .
$$


The ratio of the inertial pressure to the incremental Young's modulus, required in (2.6), is given by $\rho U_{0}^{2} / E=\left(\sigma_{0} h_{0}\right) /(T a)$. In $\S 2.4$ below we reduce (2.1), (2.2), (2.7) to a simpler system by assuming $R e, T, L, L_{1}$ and $L_{2}$ are all large.

\subsection{Numerical method}

The numerical simulation of the large-displacement fluid-structure interaction problem governed by equations (2.1) and (2.3)-(2.6) was performed with an existing fully coupled finite-element code which has previously been used in a variety of applications (Heil 2000; Heil \& White 2002). Briefly, LBB-stable quadrilateral elements (with continuous biquadratic and discontinuous bilinear representations for the velocities and the pressure, respectively; see Sani et al. 1981a,b) were used to discretize the Arbitrary Lagrangian-Eulerian form of the Navier-Stokes equations (2.1) on a body-fitted moving mesh. The mesh update in response to changes in the wall position was performed by the 'Method of Spines' (Kistler \& Scriven 1983) and all time-derivatives were discretized by the second-order backward Euler method (BDF2). Traction-free parallel outflow was imposed at the downstream end of the channel (at $x=L+L_{2}$ ); the prescribed pressure drop was imposed by applying the (pseudo-)traction boundary condition $\left(-p+u_{x}, u_{y}+v_{x}\right)^{T}=\left(-p_{u p}, 0\right)^{T}$ at $x=-L_{1}$. The variational principle (2.3) was discretized by displacement-based Hermite elements (Bogner, Fox \& Schmit 1967) and the resulting system of coupled nonlinear algebraic equations was solved by a Newton-Raphson method, using the frontal solver MA42 from the HSL2000 library to assemble and decompose the Jacobian matrices. We refer to Heil (2000) and Heil \& White (2002) for a more detailed description of the numerical method and for details of the code validation.

A steady version of the code was used to determine the system's equilibrium configurations for given values of $R e, T$ and $p_{e}$. To facilitate comparisons with the analytical results, which are based upon perturbations about the undeformed configuration, we employed a displacement-control technique to determine the external pressure $p_{e}^{(f l u s h)}(R e, T)$ for which the vertical wall displacement at the centre of the elastic segment is zero. We refer to this state as the 'flush' configuration even though the small viscous pressure drop along the elastic segment causes its upstream (downstream) ends to bulge out (collapse inwards) slightly. To determine the stability of the steady configuration we started the time-dependent simulation from the steady solution for a slightly smaller external pressure of $p_{e}=p_{e}^{(f \text { flush })}-\Delta p_{e}$ where $\left|\Delta p_{e}\right| \ll 1$. For $t \geqslant 0$, we set $p_{e}=p_{e}^{(f l u s h)}$ and followed the system's evolution. Initially, the wall performed small-amplitude oscillations about the 'flush' position and the growth (or decay) rates of these oscillations indicated the stability of the steady state.

During the early stages of the system's evolution, the largest velocity gradients occur in Stokes layers on the upper and lower channel walls. We used an appropriately refined mesh with a total of 16290 degrees of freedom (DOFs) for the simulation of the small-amplitude oscillations. To establish the mesh independence of the results, selected computations were repeated at a finer resolution of 28610 DOFs (see figure $5 b$ below). The simulation of the large-amplitude oscillations presented in $\S 4.2$ required the use of much finer meshes. The standard resolution for these computations involved 83240 DOFs and the spatial convergence was verified in a further simulation involving 160140 DOFs (see figures $12(\mathrm{~m})$ and 14 below). The time-step in the BDF2 scheme was chosen such that each period of the oscillation contained approximately 500 steps. The temporal convergence of the solutions was established by halving and doubling the time-steps in selected simulations (see figure $5 b$ ). 
The number of elements used for the discretization of the variational principle (2.3) was matched to the number of elements in the fluid domain. This resulted in a finer-than-necessary discretization of the wall equations but the resulting (modest) increase in the overall number of DOFs was more than compensated for by dramatic improvements in the efficiency of the sparse mesh-update algorithm. Computational results are given in $\S 4$ below.

\subsection{Long-wavelength, high-frequency rescaling}

For the purposes of the asymptotic analysis in $\S 3$ below, we rescale (2.1), (2.2), (2.7) to capture long-wavelength, high-frequency motions. With $\epsilon$ as defined in (2.8), we make a long-wavelength rescaling by writing

$$
x=\hat{x} / \epsilon, \quad v=\hat{v} \epsilon, \quad t=\hat{t} / \epsilon, \quad R e=\hat{R} / \epsilon, \quad T=\hat{T} / \epsilon^{2}, \quad L_{1}=\hat{L}_{1} / \epsilon, \quad L_{2}=\hat{L}_{2} / \epsilon .
$$

Then, dropping hats from the variables, we have

$$
\begin{gathered}
\nabla \cdot \boldsymbol{u}=0, \\
u_{t}+(\boldsymbol{u} \cdot \nabla) u=-p_{x}+\left(u_{y y}+\epsilon^{2} u_{x x}\right) / \hat{R}, \\
v_{t}+(\boldsymbol{u} \cdot \nabla) v=-\epsilon^{-2} p_{y}+\left(v_{y y}+\epsilon^{2} v_{x x}\right) / \hat{R}, \\
u=0, \quad v=h_{t}, \quad p=p_{e}-\frac{\hat{T} h_{x x}}{\left(1+\epsilon^{2} h_{x}^{2}\right)^{3 / 2}}+O\left(\epsilon^{2} / \hat{R}\right) \quad(y=h, \quad 0<x<1), \\
p=12\left(1+\hat{L}_{1}+\hat{L}_{2}\right) / \hat{R} \quad \text { or } q=1 \quad\left(x=-\hat{L}_{1}\right),
\end{gathered}
$$

and the remaining boundary conditions as before. The size of the viscous contribution to the normal stress is indicated in $(2.10 d)$. Setting $\delta=\hat{T}^{-1 / 2} \ll 1$, we rescale again to capture high-frequency motions with small-amplitude membrane displacements, writing

$$
t=\delta \tilde{t}, \quad h=1+\delta \tilde{h}, \quad p=\tilde{p} / \delta, \quad p_{e}=\tilde{p}_{e} / \delta, \quad \hat{R}=r^{2} / \delta,
$$

which gives (dropping tildes from the variables)

$$
\begin{aligned}
& \nabla \cdot \boldsymbol{u}=0 \\
& u_{t}+\delta(\boldsymbol{u} \cdot \nabla) u=-p_{x}+\delta^{2}\left(u_{y y}+\epsilon^{2} u_{x x}\right) / r^{2}, \\
& v_{t}+\delta(\boldsymbol{u} \cdot \nabla) v=-\epsilon^{-2} p_{y}+\delta^{2}\left(v_{y y}+\epsilon^{2} v_{x x}\right) / r^{2}, \\
& u=0, \quad v=h_{t} \quad p=\tilde{p}_{e}-h_{x x}+O\left(\epsilon^{2} \delta^{2}\right) \quad(y=1+\delta h, \quad 0<x<1), \\
& h=0 \quad(x=0,1) \text {, } \\
& p=12 \delta^{2}\left(1+\hat{L}_{1}+\hat{L}_{2}\right) / r^{2} \quad \text { or } \quad q=1 \quad\left(x=-\hat{L}_{1}\right), \\
& p=0 \quad \text { or } q=1 \quad\left(x=1+\hat{L}_{2}\right),
\end{aligned}
$$

and the remaining conditions as before. Variations in tension due to viscous stresses are then $O\left(\epsilon^{2} \delta^{3}\right)$ smaller than the mean tension, so we can safely ignore them. We now restrict attention to the limit in which $\lambda=\epsilon^{2} / \delta=O(1)$ and $r=O(1)$, and discard all terms that are $O\left(\delta^{3}\right)$, so that $(2.12 a-c)$ for example become

$$
\begin{gathered}
u_{x}+v_{y}=0, \\
u_{t}+\delta(\boldsymbol{u} \cdot \nabla) u=-p_{x}+\delta^{2} u_{y y} / r^{2}, \\
\delta \lambda\left[v_{t}+\delta(\boldsymbol{u} \cdot \nabla) v\right]=-p_{y} .
\end{gathered}
$$


Later we will make use of the vorticity equation, which reduces to

$$
\omega_{t}+\delta(\boldsymbol{u} \cdot \nabla) \omega=\delta^{2} \omega_{y y} / r^{2} \text { where } \omega=\lambda \delta v_{x}-u_{y} .
$$

We will also use the energy equation which, when integrated over the collapsible segment of the channel, can be expressed to $O\left(\delta^{2}\right)$ as

$$
\begin{aligned}
& \left(\frac{1}{2} \int_{0}^{1} \int_{0}^{h}\left(u^{2}+\lambda \delta v^{2}\right) \mathrm{d} y \mathrm{~d} x\right)_{t}+\delta\left[\int_{0}^{1} \frac{1}{2} u\left(u^{2}+\lambda \delta v^{2}\right) \mathrm{d} y\right]_{x=0}^{x=1} \\
& +\left[\int_{0}^{1} u p \mathrm{~d} y\right]_{x=0}^{x=1}+\left.\int_{0}^{1}(p v)\right|_{y=h} \mathrm{~d} x+\frac{\delta^{2}}{r^{2}} \int_{0}^{1} \int_{0}^{h} u_{y}^{2} \mathrm{~d} y \mathrm{~d} x=0 .
\end{aligned}
$$

Similar expressions can be derived for the fluid occupying $-\hat{L}_{1} \leqslant x \leqslant 0$ and $1 \leqslant$ $x \leqslant 1+\hat{L}_{2}$. In (2.15) we can identify terms corresponding respectively to the rate of change of kinetic energy, kinetic energy flux, the rate of working of pressure forces on the fluid and on the membrane, and viscous dissipation.

In $\S 3$ below we construct an asymptotic solution of (2.13) and the corresponding boundary conditions for $\delta \ll 1$, parameterized by $\hat{L}_{1}, \hat{L}_{2}, \lambda, r$ and $\tilde{p}_{e}$. The primary outcome is a stability threshold for the onset of self-excited oscillations, expressed as a critical value $r_{c 0}$ of $r$ (see $\left.(3.48 b),(3.51)\right)$. The results of the analysis are summarized in $\S 3.7$.

\section{Asymptotic analysis}

We start by expanding all variables as $\boldsymbol{u}=\boldsymbol{u}_{0}+\delta \boldsymbol{u}_{1}+\delta^{2} \boldsymbol{u}_{2}+\ldots$, etc., and introducing slow time variables $t_{i}=t \delta^{i}, i=1,2, \ldots$ For notational simplicity, $\delta \boldsymbol{u}_{1}$ will be taken to include terms between $O(\delta)$ and $O\left(\delta^{2}\right)$ in magnitude; contributions of $O\left(\delta^{1 / 2}\right)$ and $O\left(\delta^{4 / 3}\right)$ will arise later, for example. Thus at $O(1)$ in the core of the channel, $(2.13)$ plus the corresponding boundary conditions give

$$
u_{0 x}+v_{0 y}=0, \quad u_{0 t}=-p_{0 x}, \quad 0=-p_{0 y}
$$

subject to

$$
\begin{aligned}
& v_{0}=h_{0 t}, \quad p_{0}=\tilde{p}_{e}-h_{0 x x} \quad(y=1, \quad 0 \leqslant x \leqslant 1), \\
& v_{0}=0 \quad(y=0), \\
& p_{0}=0 \quad \text { or } \quad q_{0}=1 \quad\left(x=-\hat{L}_{1}\right) \text {, } \\
& p_{0}=0 \quad \text { or } \quad q_{0}=1 \quad\left(x=1+\hat{L}_{2}\right),
\end{aligned}
$$

with $h_{0}=0$ at $x=0,1$. At $O(\delta)$,

$$
u_{1 x}+v_{1 y}=0, \quad u_{1 t}+\left(\boldsymbol{u}_{0} \cdot \nabla\right) u_{0}=-p_{1 x}-u_{0 t_{1}}, \quad \lambda v_{0 t}=-p_{1 y}
$$

subject to

$$
\begin{array}{llll}
p_{1}=-h_{1 x x} & & (y=1+\delta h, \quad 0 \leqslant x \leqslant 1), \\
p_{1}=0 \quad \text { or } \quad q_{1}=0 & \left(x=-\hat{L}_{1}\right), \\
p_{1}=0 \quad \text { or } \quad q_{1}=0 & \left(x=1+\hat{L}_{2}\right),
\end{array}
$$

with $h_{1}=0$ at $x=0,1$. We anticipate that $v_{1}$ is forced by boundary layers at $y=0,1$. At $O\left(\delta^{2}\right)$,

$$
\begin{gathered}
u_{2 x}+v_{2 y}=0, \\
u_{2 t}+\left(\boldsymbol{u}_{0} \cdot \nabla\right) u_{1}+\left(\boldsymbol{u}_{1} \cdot \nabla\right) u_{0}=-p_{2 x}+\left(u_{0 y y} / r^{2}\right)-u_{1 t_{1}}-u_{0 t_{2}},
\end{gathered}
$$




$$
\lambda\left(v_{1 t}+\left(\boldsymbol{u}_{0} \cdot \nabla\right) v_{0}\right)=-p_{2 y}-\lambda v_{0 t_{1}}
$$

subject to

$$
\begin{array}{lll}
p_{2}=-h_{2 x x} & & (y=1+\delta h, \quad 0 \leqslant x \leqslant 1), \\
p_{2}=12\left(1+\hat{L}_{1}+\hat{L}_{2}\right) / r^{2} \quad \text { or } \quad q_{2}=0 \quad & \left(x=-\hat{L}_{1}\right), \\
p_{2}=0 \quad \text { or } \quad q_{2}=0 & & \left(x=1+\hat{L}_{2}\right),
\end{array}
$$

with $h_{2}=0$ at $x=0,1$ and $v_{2}$ at $y=0,1$ set by boundary layers.

We have already indicated the existence of viscous boundary layers (Stokes layers) on each wall of the channel, having thickness of $O(\delta / r)$ (so that the unsteady and viscous terms balance in (2.14), for example). To describe these layers, on the lower wall we let $y=(\delta / r) Y, u=U\left(x, Y, t, t_{1}, t_{2}, \ldots\right), v=(\delta / r) V\left(x, Y, t, t_{1}, t_{2}, \ldots\right)$, and so (2.13) gives

$$
\begin{gathered}
U_{x}+V_{Y}=0, \\
U_{t}+\delta U_{t_{1}}+\delta^{2} U_{t_{2}}+\delta(\boldsymbol{U} \cdot \nabla) U=-p_{x}+U_{Y Y}, \\
0=-p_{Y},
\end{gathered}
$$

with $U=V=0$ on $Y=0$ and error $O\left(\delta^{3}\right)$. On the upper wall, we employ a Prandtl transformation, setting

$$
\begin{gathered}
y=1+\delta h-(\delta / r) Y, \quad u=U\left(x, Y, t, t_{1}, t_{2}, \ldots\right), \\
v=\delta h_{x} U-(\delta / r) V+h_{t}+\delta h_{t_{1}}+\delta^{2} h_{t_{2}}+O\left(\delta^{3}\right),
\end{gathered}
$$

giving (3.4) again. Expanding in powers of $\delta$, (3.4) gives $\nabla \cdot \boldsymbol{U}_{i}=0$ and $p_{i Y}=0$ for $i=0,1,2$ with

$$
\begin{gathered}
U_{0 t}=-p_{0 x}+U_{0 Y Y} \\
U_{1 t}+\left(\boldsymbol{U}_{0} \cdot \nabla\right) U_{0}=-p_{1 x}+U_{1 Y Y}-U_{0 t_{1}}, \\
U_{2 t}+\left(\boldsymbol{U}_{0} \cdot \nabla\right) U_{1}+\left(\boldsymbol{U}_{1} \cdot \nabla\right) U_{0}=-p_{2 x}+U_{2 Y Y}-U_{1 t_{1}}-U_{0 t_{2}} .
\end{gathered}
$$

Again, $U_{i}=V_{i}=0$ on $Y=0$ for $i=0,1,2$.

We then assume the following time dependence:

$$
\begin{gathered}
\boldsymbol{u}_{0}=\boldsymbol{u}_{00}+\boldsymbol{u}_{01} \mathrm{e}^{\mathrm{i} \beta t}+\overline{\boldsymbol{u}}_{01} \mathrm{e}^{-\mathrm{i} \beta t}, \\
\boldsymbol{u}_{1}=\boldsymbol{u}_{10}+\boldsymbol{u}_{11} \mathrm{e}^{\mathrm{i} \beta t}+\overline{\boldsymbol{u}}_{11} \mathrm{e}^{-\mathrm{i} \beta t}+\boldsymbol{u}_{12} \mathrm{e}^{2 \mathrm{i} \beta t}+\overline{\boldsymbol{u}}_{12} \mathrm{e}^{-2 \mathrm{i} \beta t}, \\
\boldsymbol{u}_{2}=\boldsymbol{u}_{20}+\boldsymbol{u}_{21} \mathrm{e}^{\mathrm{i} \beta t}+\overline{\boldsymbol{u}}_{21} \mathrm{e}^{-\mathrm{i} \beta t}+\boldsymbol{u}_{22} \mathrm{e}^{2 \mathrm{i} \beta t}+\overline{\boldsymbol{u}}_{22} \mathrm{e}^{-2 \mathrm{i} \beta t},
\end{gathered}
$$

where bars denote complex conjugates, and the coefficients $\boldsymbol{u}_{i j}$ depend on $t_{1}, t_{2}$, etc. The frequency $\beta$ is to be determined. We now proceed by considering the following sequence of problems: 00, 01, 10,11, 20. Each problem demands a solution in the core and the boundary layers. A brief outline of the calculation below is as follows: $\boldsymbol{u}_{00}$ is the mean Poiseuille flow; $\boldsymbol{u}_{01}$ captures the largely inviscid normal modes of the system, which have oscillatory Stokes layers at each boundary; $\boldsymbol{u}_{10}$ is driven in part by a mean deflection of the membrane, and in part by steady streaming arising in the Stokes layers; and a solvability condition is applied to $\boldsymbol{u}_{11}$ to determine whether $\boldsymbol{u}_{01}$ slowly grows or decays in amplitude by extracting energy from the mean flow and losing it to viscous dissipation. The problem for $\boldsymbol{u}_{20}$ must be considered to determine $\boldsymbol{u}_{10}$ and the effect of the oscillations on the mean flux. 


\subsection{Problem 00}

The steady leading-order flow satisfies (from (3.1)) $\nabla \cdot \boldsymbol{u}_{00}=0$ and $\nabla p_{00}=\mathbf{0}$, with

$$
p_{00}=\tilde{p}_{e}-h_{00 x x} \quad(0 \leqslant x \leqslant 1),
$$

$v_{00}=0$ on $y=0$ and $y=1, p_{00}=0$ or $q_{00}=1$ at $x=-\hat{L}_{1}, p_{00}=0$ or $q_{00}=1$ at $x=1+\hat{L}_{2}$ and $h_{00}=0$ at $x=0,1$. Thus $p_{00}=0$ and

$$
h_{00}=-\frac{1}{2} \tilde{p}_{e} x(1-x) \text {. }
$$

For $\tilde{p}_{e}$ fixed, $h_{00}$ is independent of $t_{1}, t_{2}$ etc. Although we do not expect any boundarylayer structures to emerge at this order, the leading-order equations in each Stokes layer (3.6a) must be satisfied. This requires $\nabla \cdot \boldsymbol{U}_{00}=0, U_{00 Y Y}=0$ with $U_{00}=0$ on $Y=0$, so $U_{00}=\alpha_{ \pm} Y, V_{00}=0$ for some constants $\alpha_{ \pm}$. Matching to the core then demands that $\alpha_{ \pm}=0$, so $U_{00}=0$ and $u_{00}=o(1)$ as $y \rightarrow 0,1$.

While the constraints on $\boldsymbol{u}_{00}$ are inadequate to specify it fully, assuming that $\boldsymbol{u}_{00}$ is a Poiseuille flow with uniform flux $q_{00}$,

$$
u_{00}=6 q_{00} y(1-y), \quad v_{00}=0, \quad \omega_{00}=-6 q_{00}(1-2 y),
$$

enables us to construct self-consistent solutions to the problem. We proceed using this assumption, and furthermore we assume that $q_{00}$ does not vary on slow timescales. If the flux is specified either up- or downstream, $q_{00}=1$; otherwise $q_{00}$ is determined by the imposed pressure drop.

\subsection{Problem 01}

\subsubsection{Primary oscillations in the core}

The unsteady leading-order problem reveals the neutrally stable normal modes of the system, arising through a dominant balance of unsteady fluid inertia and membrane tension. Equations (3.1) give

$$
u_{01 x}+v_{01 y}=0, \quad \mathrm{i} \beta u_{01}=-p_{01 x}, \quad 0=-p_{01 y}
$$

subject to

$$
\begin{aligned}
& v_{01}=\mathrm{i} \beta h_{01}, \quad p_{01}=-h_{01 x x} \quad(y=1, \quad 0 \leqslant x \leqslant 1), \\
& v_{01}=0 \quad(y=0) \text {, } \\
& p_{01}=0 \quad \text { or } \quad q_{01}=0 \quad\left(x=-\hat{L}_{1}\right), \\
& p_{01}=0 \quad \text { or } \quad q_{01}=0 \quad\left(x=1+\hat{L}_{2}\right),
\end{aligned}
$$

with $h_{01}=0$ at $x=0,1$. Thus $p_{01}$ and $u_{01}$ are independent of $y$, and so $v_{01 y}=-u_{01 x}$ gives $v_{01}=-u_{01 x} y$. Thus $\mathrm{i} \beta h_{01}=-u_{01 x}$ in $0 \leqslant x \leqslant 1$. The motion is irrotational, with $\omega_{01}=0$. Then in $0 \leqslant x \leqslant 1,-p_{01 x x}=\mathrm{i} \beta u_{01 x}=\mathrm{i} \beta\left(-\mathrm{i} \beta h_{01}\right)=h_{01 x x x x}$, so that

$$
\mathscr{L} h_{01} \equiv h_{01 x x x}-\beta^{2} h_{01}=0 \quad(0 \leqslant x \leqslant 1) .
$$

In the upstream rigid segment, $v_{01}$ vanishing on $y=1$ demands that $u_{01}$ is uniform, so either $p_{01}=-\mathrm{i} \beta u_{01}(0)\left(x+\hat{L}_{1}\right)$ if a pressure boundary condition is imposed, implying $\left(\partial_{x}^{2}-\hat{L}_{1} \partial_{x}^{3}\right) h_{01}(0)=0$, or $u_{01}(0)=0$ if a flux boundary condition is imposed, implying $h_{01 x x x}(0)=0$. We can therefore mimic the prescribed-upstream-flux case by taking the limit $\hat{L}_{1} \rightarrow \infty$. In the downstream segment, $p_{01}=\mathrm{i} \beta u_{01}(1)\left(1+\hat{L}_{2}-x\right)$, so that $\left(\partial_{x}^{2}+\hat{L}_{2} \partial_{x}^{3}\right) h_{01}(1)=0$ (and the prescribed-downstream-flux case then corresponds to $\hat{L}_{2} \rightarrow \infty$ ). The general eigenvalue problem for the primary oscillation is therefore 
(3.12a) subject to

$$
\left(\partial_{x}^{2}-\hat{L}_{1} \partial_{x}^{3}\right) h_{01}(0)=0, \quad\left(\partial_{x}^{2}+\hat{L}_{2} \partial_{x}^{3}\right) h_{01}(1)=0, \quad h_{01}(0)=h_{01}(1)=0 .
$$

For later reference, we note that under the inner product $\langle f, g\rangle \equiv \int_{0}^{1} f g \mathrm{~d} x$, (3.12) has an adjoint solution $h_{01}^{\dagger}$ satisfying $\mathscr{L} h_{01}^{\dagger}=0$ in $0 \leqslant x \leqslant 1$,

$$
\left(1-\hat{L}_{1} \partial_{x}\right) h_{01 x}^{\dagger}(0)=0, \quad\left(1+\hat{L}_{2} \partial_{x}\right) h_{01}^{\dagger}(1)=0, \quad h_{01 x x}^{\dagger}(0)=h_{01 x x}^{\dagger}(1)=0,
$$

ensuring that $\left\langle h_{01}^{\dagger}, \mathscr{L} h_{01}\right\rangle=\left\langle\mathscr{L} h_{01}^{\dagger}, h_{01}\right\rangle$. It follows that $h_{01}^{\dagger}=h_{01 x x}$, up to an arbitrary normalization.

We write the general solution of (3.12) as

$$
h_{01}=A \phi_{01}, \quad u_{01}=A \phi_{01 x x x} /(i \beta), \quad v_{01}=i \beta A \phi_{01} y, \quad p_{01}=-A \phi_{01 x x},
$$

where $A\left(t_{1}, t_{2}, \ldots\right)$ is a slowly varying complex amplitude and $\phi_{01}(x)$ satisfies (3.12). The problem has eigenfunctions of the form

$$
\phi_{01}=A_{1} \sinh (z x)+A_{2} \cosh (z x)+A_{3} \sin (z x)+A_{4} \cos (z x),
$$

where $z=\sqrt{\beta}$ and $A_{1}, \ldots, A_{4}$ are constants. The eigenfrequencies $\beta$ form an ordered set $0<\beta_{1}<\beta_{2}<\ldots$, each satisfying

$\left|\begin{array}{cccc}0 & 1 & 0 & 1 \\ \hat{L}_{1} z & -1 & \hat{L}_{1} z & 1 \\ \sinh z & \cosh z & -\sin z & \cos z \\ \sinh z+\hat{L}_{2} z \cosh z & \cosh z+\hat{L}_{2} z \sinh z & \sin z+\hat{L}_{2} z \cos z & -\cos z+\hat{L}_{2} z \sin z\end{array}\right|=0$.

For given $\hat{L}_{1}$ and $\hat{L}_{2}$ these eigenvalues and eigenfunctions are readily determined: some examples are given in $\S 3.7$ below.

Two important identities to be used later can be derived from (3.12) using integration by parts:

$$
\begin{gathered}
{\left[\phi_{01 x x x}^{2}\right]_{0}^{1}=-\beta^{2}\left[\phi_{01 x}^{2}\right]_{0}^{1},} \\
\int_{0}^{1} \phi_{01 x x x}^{2} \mathrm{~d} x+\hat{L}_{1} \phi_{01 x x x}^{2}(0)+\hat{L}_{2} \phi_{01 x x x}^{2}(1)=\beta^{2} \int_{0}^{1} \phi_{01 x}^{2} \mathrm{~d} x .
\end{gathered}
$$

When $\hat{L}_{1}=\hat{L}_{2}, \phi_{01}(x)=\phi_{01}(1-x)$; when $\hat{L}_{2}>\hat{L}_{1}$, numerical solutions of $(3.12)$ indicate that $\phi_{01 x x x}^{2}(0)>\phi_{01 x x x}^{2}(1)$ (since the larger downstream fluid inertia suppresses the amplitude of fluxes at $x=1$ ). It then follows that $\left[\phi_{01 x}^{2}\right]_{0}^{1}>0$ under these conditions.

\subsubsection{Stokes layers}

We now turn to the boundary layers, both of which have the form (from $(3.6 a)$ )

$$
\nabla \cdot \boldsymbol{U}_{01}=0, \quad \mathrm{i} \beta U_{01}=-p_{01 x}+U_{01 Y Y}, \quad 0=-p_{01 Y},
$$

with $U_{01}=0$ on $Y=0$ and $U_{01} \rightarrow u_{01}$ as $Y \rightarrow \infty$. This has the familiar Stokes-layer solution

$$
U_{01}=u_{01}(1-E), \quad V_{01}=-u_{01 x} Y+u_{01 x} \frac{(2 / \beta)^{1 / 2}}{1+\mathrm{i}}(1-E)
$$

where $E \equiv \exp \left[-(1+\mathrm{i})(\beta / 2)^{1 / 2} Y\right]$. In the rigid segments, where $u_{01 x}=0$, the boundarylayer structure is equivalent to that arising in high-frequency Womersley flow. In the collapsible segment, however, viscous effects in the Stokes layers generate an 
oscillatory vertical flow at $O(\delta)$ which will contribute to the core-flow component $v_{11}$. In particular, at the outer edge of each boundary layer in $0 \leqslant x \leqslant 1$, we have

$$
\begin{gathered}
v_{11} \sim \frac{u_{01 x}(2 / \beta)^{1 / 2}}{r(1+\mathrm{i})} \text { as } y \rightarrow 0, \\
v_{11} \sim \mathrm{i} \beta h_{11}+h_{01 t_{1}}-\frac{u_{01 x}(2 / \beta)^{1 / 2}}{r(1+\mathrm{i})}+\left(h_{00} u_{01}\right)_{x} \quad \text { as } y \rightarrow 1
\end{gathered}
$$

where $u_{01 x}=-\mathrm{i} \beta A \phi_{01}$. The extra terms in $(3.19 b)$ arise from inverting the Prandtl transformation (3.5) (lengthy algebra has been omitted).

\subsection{Problem 10}

\subsubsection{The core flow}

The steady flow in the core at $O(\delta)$ satisfies (from (3.2)) $\boldsymbol{\nabla} \cdot \boldsymbol{u}_{10}=0, p_{10 y}=0$ plus

$$
\begin{array}{lll}
\left(\boldsymbol{u}_{01} \cdot \nabla\right) \bar{u}_{01}+\left(\overline{\boldsymbol{u}}_{01} \cdot \nabla\right) u_{01}=-p_{10 x} \\
p_{10}=-h_{10 x x} & & (0<x<1), \\
p_{10}=0 & \text { or } \quad q_{10}=0 & \left(x=-\hat{L}_{1}\right), \\
p_{10}=0 & \text { or } \quad q_{10}=0 & \left(x=1+\hat{L}_{2}\right) .
\end{array}
$$

We have assumed here that $\boldsymbol{u}_{00}$ is unidirectional and independent of $t_{1}$, as in (3.10). Equation (3.20a) shows how the oscillatory normal mode generates a steady pressure gradient through the action of Reynolds stresses. In the core, since $u_{01}$ is a function of $x$ alone,

$$
\left(\boldsymbol{u}_{01} \cdot \nabla\right) \bar{u}_{01}+\left(\overline{\boldsymbol{u}}_{01} \cdot \nabla\right) u_{01}=2|A|^{2} \phi_{01} \phi_{01 x x x} \equiv|A|^{2}\left(\phi_{01 x x x}^{2}\right)_{x} / \beta^{2},
$$

from which $p_{10}$ can be determined. If the upstream flux is prescribed, for example, then

$$
p_{10}=|A|^{2}\left[\phi_{01 x x x}^{2}(1)-\phi_{01 x x x}^{2}(x)\right] / \beta^{2} \quad(0 \leqslant x \leqslant 1),
$$

and $p_{10}$ in the upstream rigid segment is non-zero. This pressure distribution will produce a steady deflection of the membrane $h_{10}$ through $(3.20 b)$. Under pressure-driven flow, however, the induced pressure gradient is inconsistent with the homogeneous pressure conditions $(3.20 c, d)$ (provided $\hat{L}_{1}$ and $\hat{L}_{2}$ are $O(1)$ ), suggesting in this case that we require $|A|^{2}=o(1)$ to construct a self-consistent approximation. We shall see in $\S 3.6$ below that taking $|A|=O\left(\delta^{1 / 2}\right)$ allows the pressure drop due to the mean Poiseuille flow to balance the pressure drop (3.21) due to Reynolds stresses. For the present, however, we proceed assuming $|A|=O(1)$. To determine $\boldsymbol{u}_{10}$ we must await Problem $20(\S 3.5)$.

\subsubsection{The boundary layers: the generation of steady streaming}

In each boundary layer, $(3.6 b)$ implies $p_{10 Y}=0$ with

$$
\nabla \cdot \boldsymbol{U}_{10}=0, \quad\left(\boldsymbol{U}_{01} \cdot \nabla\right) \bar{U}_{01}+\left(\overline{\boldsymbol{U}}_{01} \cdot \nabla\right) U_{01}=-p_{10 x}+U_{10 Y Y},
$$

with $U_{10}=0$ on $Y=0$, where $\boldsymbol{U}_{01}$ is given by (3.18). Thus

$$
\begin{aligned}
U_{10 Y Y}=|A|^{2} \phi_{01} \phi_{01 x x x}[2(-E-\bar{E}+E \bar{E}) & +\mathrm{i}(E-\bar{E}) \\
& \left.-Y(\beta / 2)^{1 / 2}[(1+\mathrm{i}) E+(1-\mathrm{i}) \bar{E}]\right] .
\end{aligned}
$$

This has the solution, in $0 \leqslant x \leqslant 1$, 


$$
\begin{aligned}
U_{10}= & |A|^{2} \phi_{01} \phi_{01 x x x}\left[\frac{E \bar{E}-3}{\beta}+\left(\frac{1+4 \mathrm{i}}{\beta}-\frac{Y}{(1+\mathrm{i})(\beta / 2)^{1 / 2}}\right) E\right. \\
& \left.+\left(\frac{1-4 \mathrm{i}}{\beta}-\frac{Y}{(1-\mathrm{i})(\beta / 2)^{1 / 2}}\right) \bar{E}\right]+B_{ \pm} Y, \\
V_{10}= & \frac{|A|^{2}\left(\phi_{01} \phi_{01 x x x}\right)_{x}}{\beta}\left[3 Y+\frac{E \bar{E}-13}{(2 \beta)^{1 / 2}}+\left(\frac{3+2 \mathrm{i}}{(\beta / 2)^{1 / 2}}+\mathrm{i} Y\right) E+\left(\frac{3-2 \mathrm{i}}{(\beta / 2)^{1 / 2}}-\mathrm{i} Y\right) \bar{E}\right],
\end{aligned}
$$

for some constants $B_{+}$and $B_{-}$in each boundary layer (assuming $B_{ \pm}$are independent of $x$ ). It follows that

$$
U_{10} \sim|A|^{2} u_{s}+B_{ \pm} Y, \quad V_{10} \sim-|A|^{2} u_{s x}\left(Y-\frac{13}{3(2 \beta)^{1 / 2}}\right) \quad(Y \rightarrow \infty),
$$

where $u_{s}=-3 \phi_{01} \phi_{01 x x x} / \beta$ is the steady-streaming velocity outside the lower Stokes layer, generated by Reynolds stresses within the Stokes layer. The core flow driven by the streaming is discussed in $\S 3.5$ below. For later reference, the matching conditions are

$$
u_{00} \sim B_{-} r y, \quad u_{10} \sim|A|^{2} u_{s}, \quad v_{10} \sim-|A|^{2} u_{s x} y \quad(y \rightarrow 0)
$$

(so (3.10) requires $B_{-} r=6 q_{00}$, justifying the assumption $B_{-x}=0$ ), and

$$
\begin{gathered}
u_{00} \sim B_{+} r(1-y), \quad u_{10} \sim|A|^{2} u_{s}+6 q_{00} h_{00}, \\
v_{10} \sim|A|^{2} u_{s x}(1-y)+6 q_{00}(1-y) h_{00 x}
\end{gathered}
$$

as $y \rightarrow 1$. Here we have used $h_{01 x} \bar{u}_{01}+\bar{h}_{01 x} u_{01}=0$ and $u_{01 x} \bar{h}_{01}+\bar{u}_{01 x} h_{01}=0$ (given (3.14)), and we have also used (3.10) (with $6 q_{00}=B_{+} r$ ) to invert the Prandtl transformation (3.5).

\subsection{Problem 11}

The oscillatory problem in the core at $O(\delta)$ is, from $(3.2)$,

$$
\begin{gathered}
\nabla \cdot \boldsymbol{u}_{11}=0, \\
\mathrm{i} \beta u_{11}+\left(\boldsymbol{u}_{00} \cdot \nabla\right) u_{01}+\left(\boldsymbol{u}_{01} \cdot \nabla\right) u_{00}=-p_{11 x}-u_{01 t_{1}}, \\
\mathrm{i} \beta \lambda v_{01}=-p_{11 y}, \\
p_{11}(x, 1)=-h_{11 x x},
\end{gathered}
$$

where $h_{11}=0$ at $x=0,1$ and $v_{11}$ is forced by (3.19). The vorticity equation (2.14) gives

$$
\mathrm{i} \beta \omega_{11}+\left(\boldsymbol{u}_{01} \cdot \nabla\right) \omega_{00}=0 \quad \text { where } \quad \omega_{11}=-u_{11 y}+\lambda v_{01 x} .
$$

Equations (3.14) and (3.27c) imply that $p_{11}=\frac{1}{2} \beta^{2} \lambda A y^{2} \phi_{01}+P(x)$ for some $P(x)$, so $(3.27 b)$ becomes

$$
\mathrm{i} \beta u_{11}-\mathrm{i} \beta A \phi_{01}\left(u_{00}-y u_{00 y}\right)=-\frac{1}{2} \beta^{2} \lambda A \phi_{01 x} y^{2}-P_{x}-A_{t_{1}} \phi_{01 x x x} /(\mathrm{i} \beta),
$$

where we have again assumed that $u_{00}$ is axially uniform, satisfying (3.10). Then, taking an $x$ derivative of (3.29),

$$
-\mathrm{i} \beta v_{11 y}-\mathrm{i} \beta A \phi_{01 x}\left(u_{00}-y u_{00 y}\right)=-\frac{1}{2} \beta^{2} \lambda A y^{2} \phi_{01 x x}-P_{x x}+A_{t_{1}} \mathrm{i} \beta \phi_{01} .
$$


Integrating with respect to $y$, we have along $y=1$, using (3.14), (3.19)

$$
\begin{aligned}
\mathrm{i} \beta\left[-\mathrm{i} \beta h_{11}-A_{t_{1}} \phi_{01}-\frac{2 \mathrm{i} \beta A \phi_{01}(2 / \beta)^{1 / 2}}{r(1+\mathrm{i})}+\right. & \left.\frac{\mathrm{i} A}{\beta}\left(\phi_{01 x x x} h_{00}\right)_{x}\right]-2 \mathrm{i} \beta A \phi_{01 x} q_{00} \\
& =-\beta^{2} \frac{\lambda A}{6} \phi_{01 x x}-P_{x x}+\mathrm{i} \beta \phi_{01} A_{t_{1}} .
\end{aligned}
$$

Now $p_{11}=-h_{11 x x}$ at $y=1$, so $P=-\frac{1}{2} \lambda \beta^{2} A \phi_{01}-h_{11 x x}$. Thus

$$
\begin{aligned}
\mathscr{L} h_{11}=-2 \mathrm{i} \beta A_{t_{1}} \phi_{01}+\frac{2 \beta^{2} A \phi_{01}(2 / \beta)^{1 / 2}}{r(1+\mathrm{i})} & -2 \mathrm{i} \beta A \phi_{01 x} q_{00} \\
& -\frac{1}{3} \lambda \beta^{2} A \phi_{01 x x}-A\left(\phi_{01 x x x} h_{00}\right)_{x},
\end{aligned}
$$

where $\mathscr{L}$ is as defined in $(3.12 a)$.

Now in each rigid segment

$$
u_{11 x}+v_{11 y}=0, \quad \mathrm{i} \beta u_{11}=-p_{11 x}-u_{01 t_{1}}(0,1), \quad 0=-p_{11 y},
$$

so $u_{11 x}$ is a function of $x$ alone, implying $v_{11}=-u_{11 x} y$. But $v_{11}=0$ on $y=0,1$ implies $u_{11 x}=0$, so $u_{11}$ is uniform. Thus

$$
\begin{array}{ll}
p_{11}=-\left(x+\hat{L}_{1}\right)\left[\mathrm{i} \beta u_{11}(0)+u_{01 t_{1}}(0)\right] & \left(-\hat{L}_{1} \leqslant x \leqslant 0\right), \\
p_{11}=\left(1+\hat{L}_{2}-x\right)\left[\mathrm{i} \beta u_{11}(1)+u_{01 t_{1}}(1)\right] & \left(1 \leqslant x \leqslant 1+\hat{L}_{2}\right),
\end{array}
$$

implying that $h_{11}$ satisfies the homogeneous boundary conditions given in $(3.12 b)$. Considering $\left\langle\phi_{01}^{\dagger}, \mathscr{L} h_{11}\right\rangle$ (see (3.13) for the definition of the adjoint $\phi_{01}^{\dagger}$ ), (3.32) yields the following solvability condition:

$$
\begin{aligned}
0=\left\langle\phi_{01}^{\dagger}, \phi_{01}\right\rangle\left(\frac{2 \beta^{2} A(2 / \beta)^{1 / 2}}{r(1+\mathrm{i})}\right. & \left.-2 \mathrm{i} \beta A_{t_{1}}\right)-2 \mathrm{i} \beta A q_{00}\left\langle\phi_{01}^{\dagger}, \phi_{01 x}\right\rangle \\
& -\frac{1}{3} \lambda \beta^{2} A\left\langle\phi_{01}^{\dagger}, \phi_{01 x x}\right\rangle-A\left\langle\phi_{01}^{\dagger},\left(\phi_{01 x x x} h_{00}\right)_{x}\right\rangle .
\end{aligned}
$$

Writing $A=\mathscr{A} \mathrm{e}^{\mathrm{i} \theta}$, where $\mathscr{A}$ and $\theta$ are real, so that $A_{t_{1}} / A=\left(\mathscr{A}_{t_{1}} / \mathscr{A}\right)+\mathrm{i} \theta_{t_{1}}$, we can separate real and imaginary parts of (3.35) to obtain

$$
\begin{gathered}
\theta_{t_{1}}=\frac{\lambda}{6} \beta \frac{\left\langle\phi_{01}^{\dagger}, \phi_{01 x x}\right\rangle}{\left\langle\phi_{01}^{\dagger}, \phi_{01}\right\rangle}-\frac{(\beta / 2)^{1 / 2}}{r}+\frac{\left\langle\phi_{01}^{\dagger},\left(\phi_{01 x x x} h_{00}\right)_{x}\right\rangle}{2 \beta\left\langle\phi_{01}^{\dagger}, \phi_{01}\right\rangle} \\
\frac{\mathscr{A}_{t_{1}}}{\mathscr{A}}=-\frac{(\beta / 2)^{1 / 2}}{r}-q_{00} \frac{\left\langle\phi_{01}^{\dagger}, \phi_{01 x}\right\rangle}{\left\langle\phi_{01}^{\dagger}, \phi_{01}\right\rangle} .
\end{gathered}
$$

If we look for purely oscillatory solutions, for which $\theta_{t_{1}}=\beta_{1}$ and $A_{t_{1}}=0$, we obtain the correction in frequency

$$
\beta_{1}=-\frac{\lambda \beta}{6} \frac{\int_{0}^{1} \phi_{01 x x}^{2} \mathrm{~d} x}{\int_{0}^{1} \phi_{01 x}^{2} \mathrm{~d} x}-\frac{(\beta / 2)^{1 / 2}}{r}-\frac{\tilde{p}_{e}}{4} \frac{\int_{0}^{1} x(1-x) \phi_{01 x x x}^{2} \mathrm{~d} x}{\int_{0}^{1} \phi_{01 x}^{2} \mathrm{~d} x}
$$

(which has contributions due to the effects of the finite length of the membrane, viscosity and the mean deflection of the membrane respectively) and the critical 
scaled Reynolds number at which the primary oscillations are neutrally stable

$$
r_{c}=-\left(\frac{\beta}{2}\right)^{1 / 2} \frac{\left\langle\phi_{01}^{\dagger}, \phi_{01}\right\rangle}{q_{00}\left\langle\phi_{01}^{\dagger}, \phi_{01 x}\right\rangle}=\frac{(2 \beta)^{1 / 2}}{q_{00}} \frac{\int_{0}^{1} \phi_{01 x}^{2} \mathrm{~d} x}{\left[\phi_{01 x}^{2}\right]_{0}^{1}}
$$

As indicated at the end of $\S 3.2 .1$, we expect $\left[\phi_{01 x}^{2}\right]_{0}^{1}>0$ when $\hat{L}_{2}>\hat{L}_{1}$, which is a necessary condition for $r_{c}$ to be well-defined. Since

$$
\frac{\mathscr{A}_{t_{1}}}{\mathscr{A}}=\left(\frac{\beta}{2}\right)^{1 / 2}\left(\frac{1}{r_{c}}-\frac{1}{r}\right),
$$

we see that oscillations grow in amplitude when $r>r_{c}$.

Appendix B shows that (3.38) has a direct interpretation in terms of energy: when $r=r_{c}$, then $3 \mathscr{D}_{S}=2 \mathscr{F}$, where $\mathscr{F}$ is the net flux of kinetic energy extracted from the mean flow and $\mathscr{D}_{S}$ is the rate of viscous energy dissipation in the Stokes layers. Further implications of (3.38) are discussed in $\S 3.7$ below. Meanwhile we proceed to Problem 20 in order to determine $q_{00}$ in (3.38).

\subsection{Problem 20}

In the core, this problem is governed by (from (3.3)) $\nabla \cdot \boldsymbol{u}_{20}=0$,

$$
\begin{gathered}
\left(\boldsymbol{u}_{00} \cdot \nabla\right) u_{10}+\left(\boldsymbol{u}_{10} \cdot \nabla\right) u_{00}+\left(\boldsymbol{u}_{01} \cdot \nabla\right) \bar{u}_{11}+\left(\overline{\boldsymbol{u}}_{01} \cdot \nabla\right) u_{11} \\
+\left(\boldsymbol{u}_{11} \cdot \nabla\right) \bar{u}_{01}+\left(\overline{\boldsymbol{u}}_{11} \cdot \nabla\right) u_{01}=-p_{20 x}+\left(u_{00 y y} / r^{2}\right)-u_{10 t_{1}}, \\
\lambda\left[\left(\boldsymbol{u}_{00} \cdot \nabla\right) v_{00}+\left(\boldsymbol{u}_{01} \cdot \nabla\right) \bar{v}_{01}+\left(\overline{\boldsymbol{u}}_{01} \cdot \nabla\right) v_{01}\right]=-p_{20 y},
\end{gathered}
$$

subject to $p_{20}=-h_{20 x x}$ in $0 \leqslant x \leqslant 1$,

$$
p_{20}\left(-\hat{L}_{1}, y\right)=\left(12 / r^{2}\right)\left(1+\hat{L}_{1}+\hat{L}_{2}\right), \quad p_{20}\left(1+\hat{L}_{2}, y\right)=0,
$$

with $h_{20}=0$ at $x=0,1$. The corresponding vorticity equation is

$$
\omega_{10 t_{1}}+\left(\boldsymbol{u}_{00} \cdot \nabla\right) \omega_{10}+\left(\boldsymbol{u}_{01} \cdot \nabla\right) \bar{\omega}_{11}+\left(\overline{\boldsymbol{u}}_{01} \cdot \nabla\right) \omega_{11}+\left(\boldsymbol{u}_{10} \cdot \nabla\right) \omega_{00}=\omega_{00 y y} / r^{2}
$$

$\omega_{01}=0$ as the primary oscillation is irrotational in the core. Now $\omega_{11}=(\mathrm{i} / \beta)\left(\boldsymbol{u}_{01}\right.$. $\nabla) \omega_{00}$ (see (3.28)), so we may rewrite two terms in (3.41) as

$$
\left(\boldsymbol{u}_{01} \cdot \nabla\right) \bar{\omega}_{11}+\left(\overline{\boldsymbol{u}}_{01} \cdot \nabla\right) \omega_{11}=\left(\boldsymbol{u}_{d} \cdot \nabla\right) \omega_{00}
$$

where $\boldsymbol{u}_{d}=(\mathrm{i} / \beta)\left[\left(\overline{\boldsymbol{u}}_{01} \cdot \nabla\right) \boldsymbol{u}_{01}-\left(\boldsymbol{u}_{01} \cdot \nabla\right) \overline{\boldsymbol{u}}_{01}\right]$ is the Stokes drift velocity due to the primary oscillations (Riley 2001). Since $\boldsymbol{u}_{01}$ takes the simple form given in (3.14) however, $\boldsymbol{u}_{d}=\mathbf{0}$, and so (3.41) reduces to

$$
\omega_{10 t_{1}}+\left(\boldsymbol{u}_{00} \cdot \nabla\right) \omega_{10}+\left(\boldsymbol{u}_{10} \cdot \nabla\right) \omega_{00}=0
$$

since $\omega_{00 y y}=0$ (from (3.10)). This equation is not sufficient to find $\omega_{10}$ and $\boldsymbol{u}_{10}$, however. Instead, by retaining the viscous term $\delta \omega_{10 y y} / r^{2}$ in (3.43) we can obtain a closed system from which $\boldsymbol{u}_{10}$ can be computed. Important aspects of this flow are described in Appendix A. It is shown there that $\boldsymbol{u}_{10}$ has two components: a component that is antisymmetric about $y=\frac{1}{2}$ and that scales with $\tilde{p}_{e}$, related to sideways displacement of streamlines by the steady indentation of the membrane due to the external pressure (3.9); and a symmetric component generated partly by membrane indentation and partly by steady-streaming effects through (3.26). The former flow vanishes at $x=0, x=1$. The latter has viscous boundary layers of 
thickness $O\left(\delta^{1 / 3}\right)$ at each wall, with a core flow of magnitude $O\left(\delta^{1 / 3}|A|^{2}\right)$ (due to steady streaming) and $O\left(\delta^{1 / 3} \tilde{p}_{e}\right)$ (due to indentation) that extends into the downstream rigid segment.

\subsection{The flux-amplitude relation}

As indicated in $\$ 3.3 .1$, under pressure-driven flow the steady pressure drop due to oscillatory Reynolds stresses (3.21) cannot be accommodated by the pressure drop due to the mean Poiseuille flow (the term $u_{00 y y} / r^{2}$ in (3.40a)) if $|A|=O(1)$. Instead, we assume that $|A|^{2}=O(\delta)$. Then the terms in (3.21) are relegated to Problem 20, and $(3.40 a)$ reduces to

$$
u_{10 t_{1}}+\left(|A|^{2} / \delta \beta^{2}\right)\left(\phi_{01 x x x}^{2}\right)_{x}=-p_{20 x}-\left(12 q_{00} / r^{2}\right),
$$

where $p_{20}$ satisfies the boundary conditions given in $(3.40 c)$. The steady-streaming flow interacting with the mean flow gives at leading order in the core (from (A 3))

$$
\left(\boldsymbol{u}_{00} \cdot \nabla\right) u_{10}+\left(\boldsymbol{u}_{10} \cdot \nabla\right) u_{00}=3 q_{00} q_{10 x}=O\left(\tilde{p}_{e} \delta^{1 / 3},|A|^{2} \delta^{1 / 3}\right) .
$$

Indentation effects are therefore likely to contribute first to (3.44); we assume here that $\tilde{p}_{e}=O(1)$ so that they may formally be neglected. It follows that for neutrally stable oscillations, for which $u_{10 t_{1}}=0, \mathscr{A}_{t_{1}}=0$

$$
p_{20}= \begin{cases}12\left(1+\hat{L}_{1}+\hat{L}_{2}-q_{00}\left(\hat{L}_{1}+x\right)\right) / r^{2} & \left(-\hat{L}_{1} \leqslant x \leqslant 0\right) \\ -\left(|A|^{2} / \delta \beta^{2}\right) \phi_{01 x x x}^{2}-\left(12 q_{00} x / r^{2}\right)+C & (0 \leqslant x \leqslant 1) \\ 12 q_{00}\left(1+L_{2}-x\right) / r^{2} & \left(1 \leqslant x \leqslant 1+L_{2}\right)\end{cases}
$$

for some constant $C$. Imposing continuity of pressure at $x=0, x=1$ gives (using (3.16))

$$
q_{00}=1-\frac{|A|^{2}}{\delta \beta^{2}} \frac{r^{2}\left[\phi_{01 x x x}^{2}\right]_{0}^{1}}{12\left(1+\hat{L}_{1}+\hat{L}_{2}\right)} \equiv 1+\frac{|A|^{2}}{\delta} \frac{r^{2}\left[\phi_{01 x}^{2}\right]_{0}^{1}}{12\left(1+\hat{L}_{1}+\hat{L}_{2}\right)} .
$$

As described in $\$ 3.2 .1$, computations indicate that $\left[\phi_{01 x}^{2}\right]_{0}^{1}>0$ when $\hat{L}_{2}>\hat{L}_{1}$. Equation (3.47) therefore shows that neutrally stable, small-amplitude oscillations increase $q_{00}$ above unity.

Equation (3.47) can be derived independently by examining the energy budget for neutrally stable, small-amplitude oscillations (see Appendix B). This calculation shows that for such oscillations the increase in flux in (3.47) provides sufficient viscous dissipation in the core flow to balance exactly one third of the flux of kinetic energy extracted from the mean flow (with dissipation in Stokes layers accounting for the remaining two thirds). Furthermore, combining (3.38) and (3.47) gives

$$
r_{c}\left[1+\frac{|A|^{2}}{\delta} \frac{r_{c}^{2}\left[\phi_{01 x}^{2}\right]_{0}^{1}}{12\left(1+\hat{L}_{1}+\hat{L}_{2}\right)}\right]=r_{c 0}, \quad r_{c 0} \equiv(2 \beta)^{1 / 2} \int_{0}^{1} \phi_{01 x}^{2} \mathrm{~d} x /\left[\phi_{01 x}^{2}\right]_{0}^{1},
$$

implying that the branch of neutrally stable oscillatory solutions bifurcates subcritically from $r=r_{c 0}$.

In practice, however, this neutrally stable solution branch will be unstable, and we are unlikely to see neutrally stable oscillations. Instead, over slow timescales with $t_{1}=O(1)$, while the amplitude evolves according to (3.39), $q_{00}$ will remain constant ( since $q_{00 t_{1}}=0$, see $\S 3.3 .1$ ), at a value set by initial transients. The imbalance between the steady pressure gradient generated by the oscillations and the steady viscous pressure drop can be accommodated by slow acceleration of a uniform plug-flow 


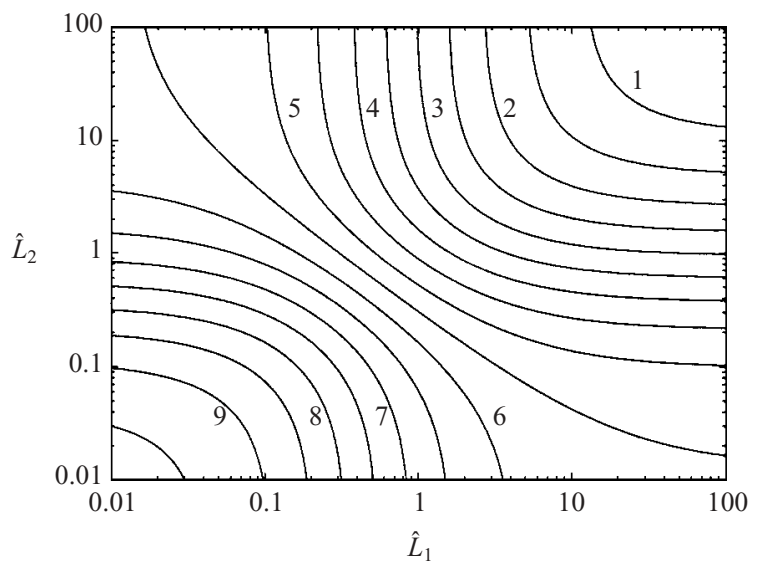

FIGURE 2. Contours of dimensionless frequency $\beta$ (with values indicated) are plotted as a function of $\hat{L}_{1}$ and $\hat{L}_{2}$. Contours are in increments of 0.5 .

contribution $q_{10}\left(t_{1}\right) \hat{\boldsymbol{x}}$ to $\boldsymbol{u}_{10}$. We must in this case retain the term $u_{10 t_{1}}$ in (3.44), which gives

$$
q_{00}=1+\frac{r^{2}}{12}\left[\frac{\left(|A|^{2} / \delta\right)\left[\phi_{01 x}^{2}\right]_{0}^{1}}{1+\hat{L}_{1}+\hat{L}_{2}}-q_{10 t_{1}}\right] .
$$

This being the case, over moderate timescales the present calculation cannot distinguish between sub- and supercritical bifurcations, since $q_{00}$ is not strongly coupled to $|A|$. Furthermore, even when $\tilde{p}_{e}=O(1)$ the effect of membrane indentation on mean energy and volume fluxes is likely to be significant (being an $O\left(\delta^{1 / 3}\right)$ effect), complicating the picture further; we do not pursue this here, however.

\subsection{Summary of asymptotic results}

We have constructed an asymptotic expansion of (2.13) and its boundary conditions in powers of $\delta=\left(\epsilon^{2} T\right)^{-1 / 2} \ll 1$ (with $\epsilon$ and $T$ as defined in (2.8)), assuming the membrane length, Reynolds number and membrane tension satisfy

$$
1 \ll L \equiv \frac{1}{\epsilon}=\frac{1}{(\lambda \delta)^{1 / 2}} \ll R e=\frac{r^{2}}{\lambda^{1 / 2} \delta^{3 / 2}} \ll T=\frac{1}{\lambda \delta^{3}},
$$

assuming $\lambda=\epsilon^{3} T^{1 / 2}$ and $r=R e^{1 / 2} T^{-1 / 4}$ are $O(1)$. We have also assumed that the rigid parts of the system have lengths comparable to the membrane (so that $\hat{L}_{1}=\epsilon L_{1}$ and $\hat{L}_{2}=\epsilon L_{2}$ are both $O(1)$ ) and that the external pressure $\tilde{p}_{e}=\delta p_{e}=O(1)$ (where $p_{e}$ is defined in (2.8)), ensuring that membrane deflections remain small.

At leading order $(\S \S 3.1,3.2)$, the flow has two independent contributions: a mean Poiseuille flow with uniform flux $q_{00}$; and a neutrally stable oscillation, which is the lowest-frequency normal mode of the system, with membrane deflections balancing the unsteady inertia of the fluid in the entire channel. The normal mode has dimensional frequency $U_{0} f / a$ where $f=\epsilon^{2} T^{1 / 2} \beta\left(\hat{L}_{1}, \hat{L}_{2}\right)$, so that the corresponding Strouhal number $S t=\epsilon / \delta=(\lambda / \delta)^{1 / 2}$ is large. A leading-order estimate of $\beta$ is given by the roots of (3.15b). Figure 2 shows that $\beta$ falls as either $\hat{L}_{1}$ or $\hat{L}_{2}$ increases, because of the increase in fluid inertia in the rigid parts of the system. 


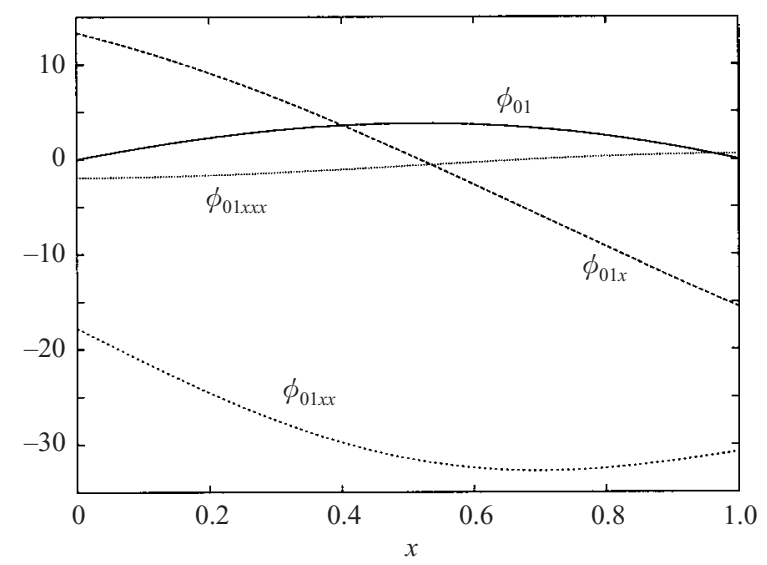

FIGURE 3. The eigenfunction $\phi_{01}(x)$ and its derivatives for $\hat{L}_{1}=0.5, \hat{L}_{2}=3$.

To illustrate this, we consider $\hat{L}_{1}=0.5, \hat{L}_{2}=3$, for which $\beta \approx 4.2915$. The corresponding eigenfunction $\phi_{01}(x)$ (see (3.14)) is shown in figure 3. Since we are considering the lowest-frequency mode, the membrane displacement $\phi_{01}$ has a single half-wavelength disturbance, with a markedly asymmetrical pressure distribution $-\phi_{01 x x}$. Correspondingly, $\left|\phi_{01 x x x}(0)\right|>\left|\phi_{01 x x x}(1)\right|$, since the larger downstream inertia suppresses velocity fluctuations, and thus $\left|\phi_{01 x}(1)\right|>\left|\phi_{01 x}(0)\right|$ (consistent with (3.16a)).

At the following order $(\S \S 3.3,3.4)$, the mean flow and the primary oscillation interact: differences in kinetic energy fluxes at either end of the membrane allow the oscillation to extract energy from the mean flow, while energy is dissipated largely in Stokes layers running along the lateral boundaries of the entire channel. A solvability condition was imposed on the component $\delta \boldsymbol{u}_{11} \mathrm{e}^{\mathrm{i} \beta t}$, giving an improved estimate of the frequency, namely $\beta+\delta \beta_{1}$ with $\beta_{1}$ given by (3.37). Increasing $\tilde{p}_{e}$ indents the membrane and causes oscillations to become slower, whereas increasing $r$ causes their frequency to rise. The solvability condition also provided an estimate (3.38) of the critical Reynolds number at which oscillations are neutrally stable. This was dependent on the flux $q_{00}$, which (under pressure-driven flow) was subsequently shown to be amplitude-dependent (see (3.47)), since Reynolds stresses due to the primary oscillation generate a steady pressure gradient which must accommodated by changing the viscous pressure drop of the mean flow. At sufficiently small amplitudes, however, we may take $q_{00} \approx 1$ and then we can use the prediction $r \approx r_{c 0}\left(\hat{L}_{1}, \hat{L}_{2}\right)$ defined in $(3.48 b)$, giving the stability threshold

$$
R e \approx r_{c 0}^{2} T^{1 / 2}
$$

which is well-defined for $\hat{L}_{2}>\hat{L}_{1}$. The dependence of flux on amplitude (3.48a) implies that, in principle, the basic state loses stability to self-excited oscillations with increasing $R e$ via a subcritical Hopf bifurcation when $\left|p_{e}\right|$ is sufficiently small, although transient effects are likely to obscure this behaviour.

Figure 4 plots $r_{c 0}$ against $\hat{L}_{1}\left(<\hat{L}_{2}\right)$ for a range of values of $\hat{L}_{2}$. When $\hat{L}_{1}=0.5$, $\hat{L}_{2}=3$, for example, $r_{c 0} \approx 3.469$. As $\hat{L}_{1} \rightarrow \hat{L}_{2}-$, the eigenfunction $\phi_{01}$ becomes symmetric about $x=\frac{1}{2}$, the denominator of $(3.48 b)$ falls to zero and $r_{c 0}$ rises to infinity. Increasing $\hat{L}_{2}$ increases the up-down asymmetry in $\phi_{01}$ and reduces $r_{c 0}$. The flow is therefore destabilized by maximizing the difference between $\hat{L}_{2}$ and $\hat{L}_{1}$. 


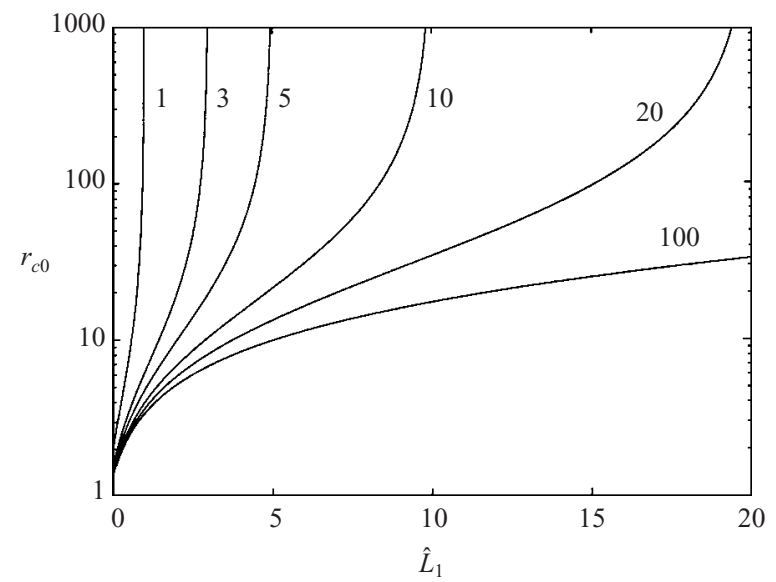

FIGURE 4. $r_{c 0}$ versus $\hat{L}_{1}$ with $\hat{L}_{2}=1,3,5,10,20$ and 100 .

\section{Computational results}

We now present results from the numerical simulations of the full problem taking $L_{1}=5, L=10$ and $L_{2}=30$ (corresponding to $\hat{L}_{1}=0.5, \hat{L}_{2}=3$ and $\epsilon=0.1$ ). In this section, dimensionless spatial variables $x, y$ are scaled on the channel width $a$.

\subsection{Small-amplitude oscillations}

Figure 5 shows a plot of the vertical wall displacement at the centre of the elastic membrane versus time for $R e=500$ at a wall tension of $T=10^{5}$ (corresponding to $\delta \approx 0.0316$ and $\lambda=0.316$ ). A steady solution for which the flexible segment bulged out slightly (so that $d^{(y)}(\zeta=5)=10^{-3}$ ) was used as the initial condition. For $t \geqslant 0$, the external pressure is set to $p_{e}=p_{e}^{(f l u s h)}=1.40133$ and the wall performs a damped oscillation about its 'flush' position. To determine the period and decay rate of the oscillation, we used the Levenberg-Marquardt algorithm to fit the computational data to the function

$$
d_{f i t}(t)=D_{0}+\mathrm{e}^{\Lambda t}\left(D_{c} \cos (2 \pi t / \mathscr{T})+D_{s} \sin (2 \pi t / \mathscr{T})\right) .
$$

The best fit to the computational data is represented by the dashed line in figure 5 . Given that the oscillation was initiated by an impulsive start and that no attempt was made to match the initial wall shape to the appropriate eigenmode, the agreement between the best fit and the computational data is extremely good. A more detailed comparison shows that the computational data do contain transient components of higher frequencies but their amplitude is so small that they cannot be detected in figure 5. These higher harmonics can be detected more easily in plots of other quantities such as the fluid pressure; see, for example, figure 8 below.

In the absence of oscillation, the pressure drop $p_{u p}=1.08$ between the upstream and downstream ends of the channel drives a steady flow with flux $q_{s} \approx 1$ through the slightly deformed channel. Figure 6 illustrates the flow fields (instantaneous streamlines and contours of the pressure) at two characteristic moments during a small-amplitude oscillation. At $t=0.08$ (figure $6 a$ ) the rapid inward motion of the wall injects a volume flux $q_{\text {wall }}=-\int_{0}^{L} h_{t} \mathrm{~d} x=0.0873$ into the channel. Approximately $\frac{3}{4}\left(\frac{1}{4}\right)$ of this flux is directed upstream (downstream) against (in) the direction of the steady flux $q_{s}$. This leads to a reduction (increase) in the flow rate in the upstream 

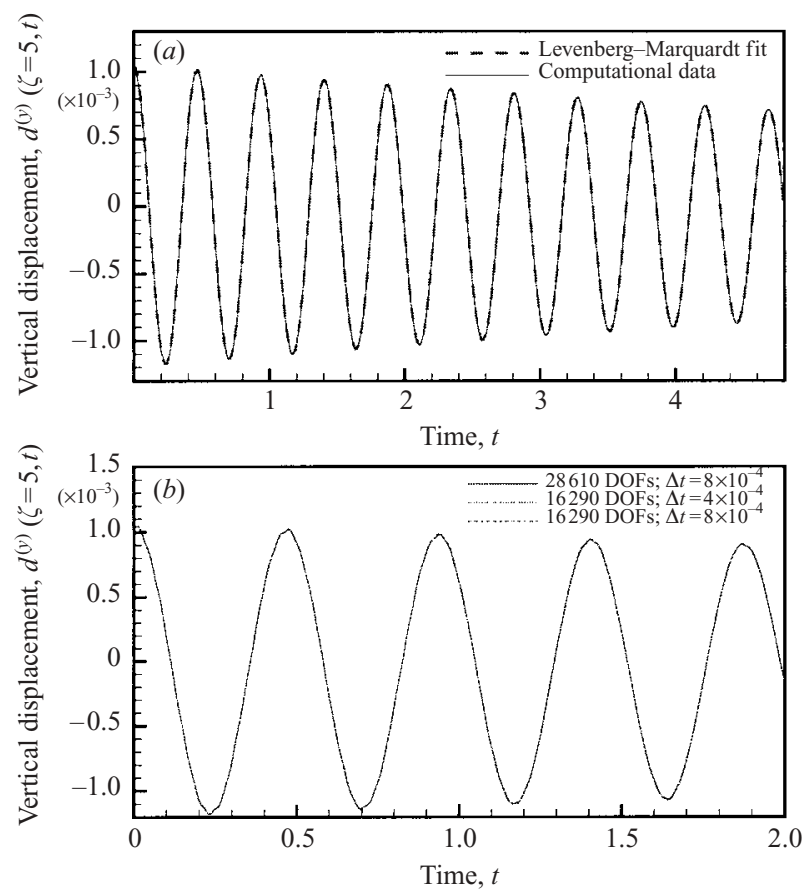

FIGURE 5. The vertical wall displacement at the centre of the elastic membrane vs. time for $R e=500, T=10^{5}$ and $p_{e}=p_{e}^{(f l u s h)}=1.40133$. The wall oscillates about the 'flush' position. (a) Comparison of the computational data (solid line) and the best fit to a damped harmonic oscillation (dashed line). (b) Results of the spatial and temporal convergence tests.

(a)

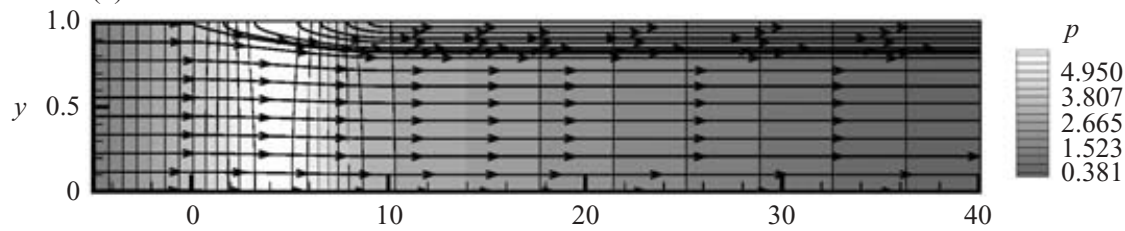

(b)

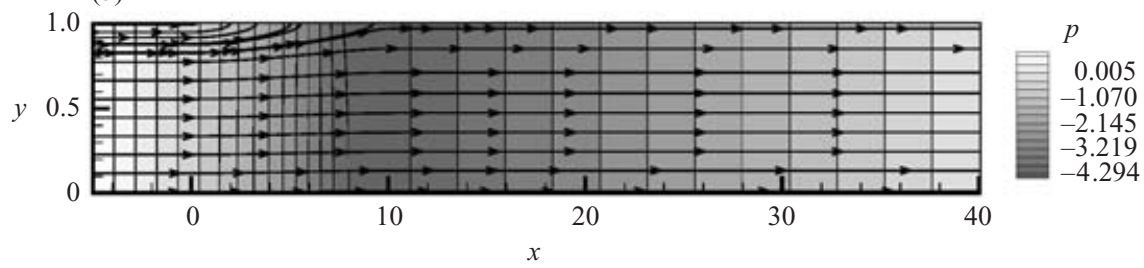

FIGURE 6. Pressure contours and instantaneous streamlines during the two characteristic phases of the small-amplitude oscillation. (a) $t=0.08$, the wall moves inwards. $(b) t=0.32$, the wall moves outwards. $R e=500, T=10^{5}$ and $p_{e}=1.40133$.

(downstream) rigid segment. The situation is reversed at $t=0.32$ when the wall moves outwards, $h_{t}>0$, as shown in figure $6(b)$.

The pressure contours in figure 6 confirm that the pressure is approximately uniform across the channel width (consistent with $(2.13 c)$, which shows that cross-stream 


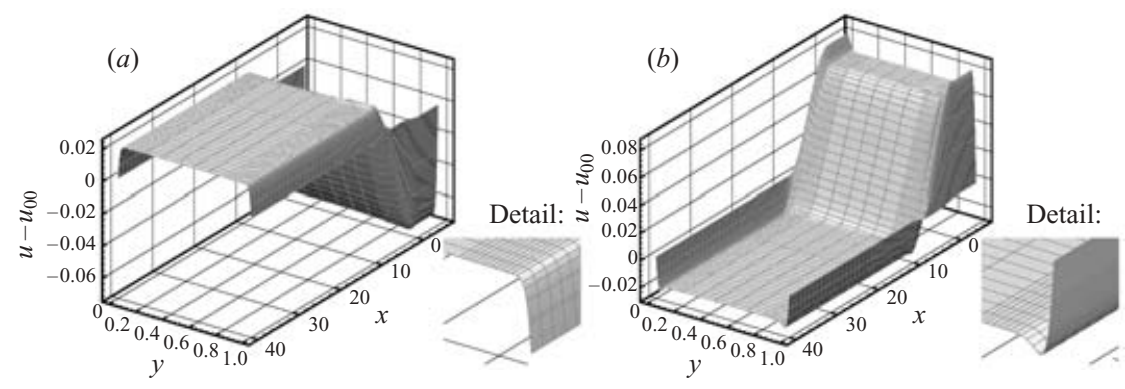

FIGURE 7. Plots of the axial velocity perturbation $u-u_{00}$ during the two characteristic phases of the small-amplitude oscillation. (a) $t=0.08$, the wall moves inwards. (b) $t=0.32$, the wall moves outwards. $R e=500, T=10^{5}$ and $p_{e}=1.40133$. Shaded surfaces: results obtained with the standard spatial resolution. Wire mesh plot: results obtained with a finer spatial resolution (containing twice as many elements in the boundary layers). The insets show details of the velocity profiles near $x=40, y=1$.

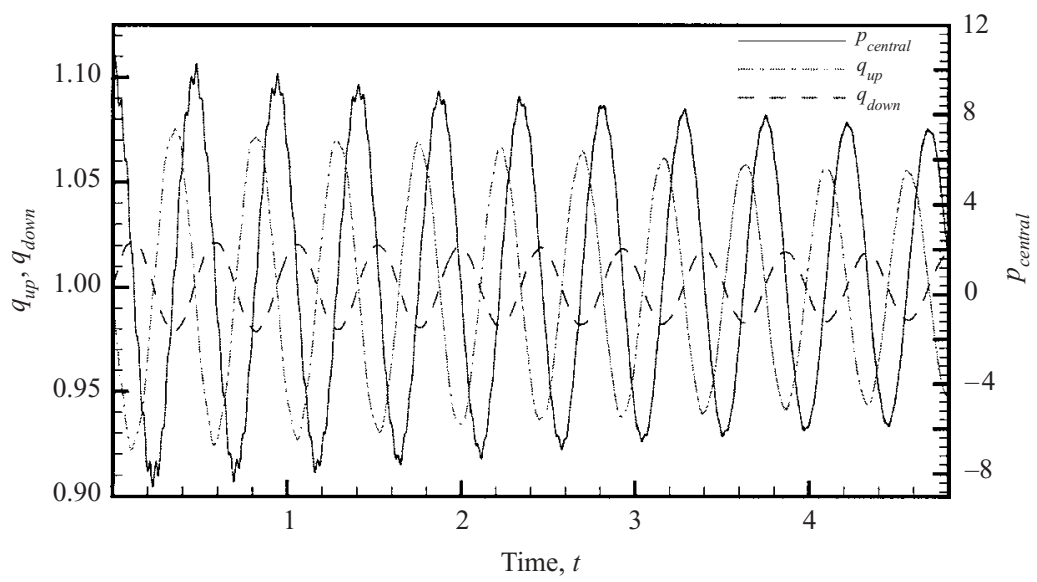

FiguRE 8 . The pressure, $p_{\text {central, }}$, at a central point in the fluid domain (at $x=4.81, y=0.39$ ) and the fluxes in the upstream and downstream rigid sections, respectively. $p_{\text {central }}$ provides a measure of the pressure drops through the rigid sections. $R e=500, T=10^{5}$ and $p_{e}=1.40133$.

pressure gradients should be at most $O(\delta)$ ). They also show that the oscillatory pressure variations dominate the primary pressure drop $p_{u p}$.

To illustrate the character of the axial velocity perturbation, figure 7 shows plots of $u_{\text {perturb }}:=u-u_{00}$ (where $u_{00}$ represents the Poiseuille flow of $(3.10)$ with $q_{00}=$ 1) at the same two instants. Inside a central core region $u_{\text {perturb }}$ is approximately independent of $y$. Thin Stokes layers join the core flow to the no-slip walls and generate a characteristic Womersley velocity profile. The different amplitudes of the axial velocity perturbations in the rigid upstream and downstream parts of the channel (approximately 1:3) reflect the greater inertia of the fluid in the downstream section. This flow structure matches closely predictions of the leading-order asymptotics ( $\$ \S 3.1$, 3.2) through the terms $u_{10}$ (describing plug flow in the core) and $U_{10}$ (in the Stokes layers).

Figure 8 shows the time history of the fluxes in the upstream and downstream rigid segments; $q_{u p, \text { down }}=\int_{0}^{1} u\left(x=-L_{1}, L+L_{2}\right) \mathrm{d} y$ and the fluid pressure $p_{\text {central }}$ at a central point $(x=4.81$ and $y=0.39)$ in the fluid domain. Since the pressures at 

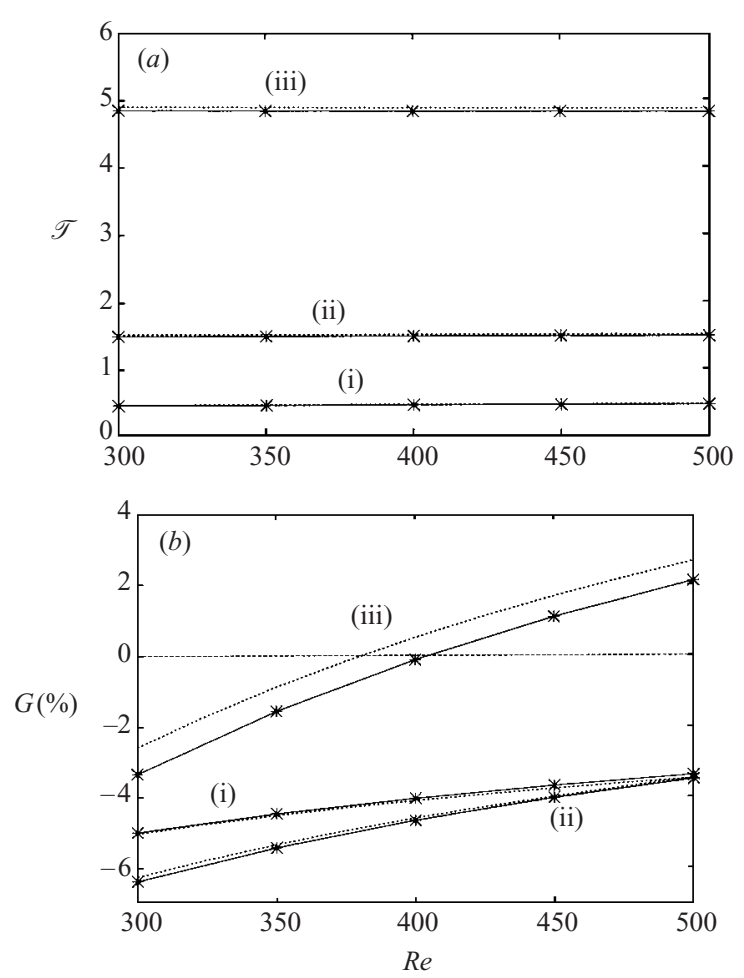

Figure 9. (a) Period $\mathscr{T}$ and $(b)$ growth rate $G=\exp (\Lambda \mathscr{T})-1$ (in percent) versus $R e$, comparing asymptotics (dashed) and computations (symbols connected by straight lines), for (i) $T=10^{5}, p_{e}=1.40133$, (ii) $T=10^{4}, p_{e}=0.840807$ and (iii) $T=10^{3}, p_{e}=1.40148$.

the upstream and downstream ends of the channel are held at fixed values, $p_{\text {central }}$ provides a measure of the pressure drop in the rigid segments; $q_{u p}$ and $q_{d o w n}$ oscillate about a mean value of $q_{\text {mean }} \approx q_{s}$ and are out of phase with each other. The phase difference between the fluxes and the driving pressure drops is close to $90^{\circ}$, confirming that the oscillatory flow is dominated by inertial effects. The relative magnitudes and phases of the upstream and downstream fluxes is consistent with the flux distribution $\phi_{01 x x x}$ given in figure 3 ; again we see that the downstream flux is smaller because of the larger fluid inertia in the downstream rigid segment.

We performed a large number of such simulations for a range of Reynolds numbers $(R e=300,350,400,450$ and 500$)$ and tensions $\left(T=10^{5}, 10^{4}\right.$ and $\left.10^{3}\right)$. In all cases, the computations covered at least 10 periods of the oscillation. The periods and growth rates were determined by a fit of the midpoint displacement data to the damped oscillation (4.1). Figure 9 compares these quantities to the corresponding asymptotic predictions: for the latter, the period was determined from the frequency of the normal mode $\beta$ (see (3.15b)) with the correction (3.37); the growth rate per period was determined from (3.36) with $q_{00}=1$. The asymptotics predict critical Reynolds numbers (see (3.51)) of approximately $12.034 T^{1 / 2}$ for $\hat{L}_{1}=0.5$ and $\hat{L}_{2}=3$, i.e. $(380.5,1203,3805)$ for $T=\left(10^{3}, 10^{4}, 10^{5}\right)$. The agreement between numerics and asymptotics is excellent for $T=10^{5}$ and $T=10^{4}$ (for which we have $\delta=0.0316$ and 0.1 , respectively), although oscillations decay in these cases; at a relatively small tension of $T=10^{3}$ (for which $\delta=0.316$ ), however, positive growth rates are both predicted and observed, and the relative discrepancies for the period and critical 


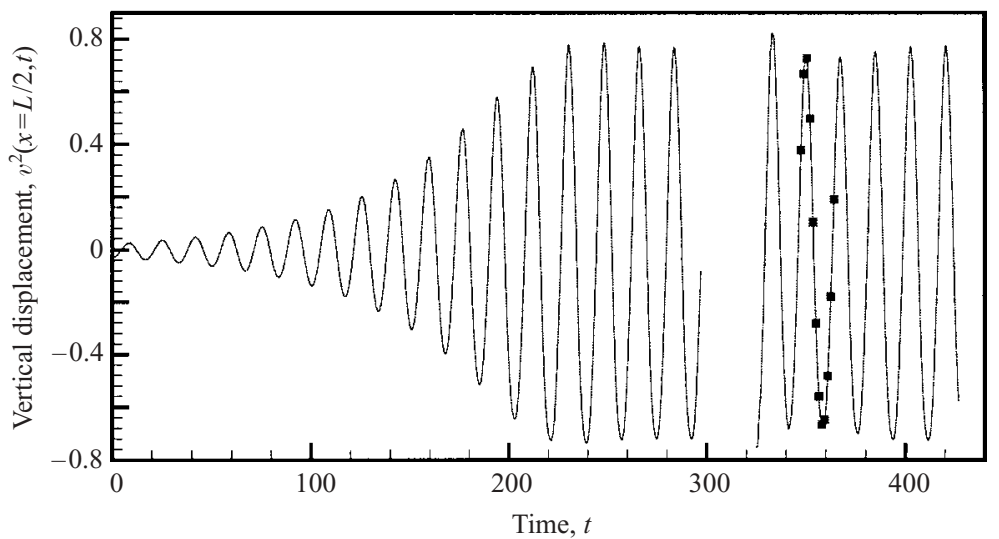

FiguRE 10. The vertical wall displacement at the centre of the membrane vs. time for $R e=450, T=10^{2}$ and $p_{e}=p_{e}^{(f l u s h)}=0.96677$. The two halves of the simulation were performed with different spatial resolutions. The symbols indicate the times at which the flow fields are documented in figure 12.

Reynolds number are approximately $1 \%$ and $6 \%$, respectively. Since none of the small-amplitude oscillations seen in computations were perfectly periodic, we were unable to detect the amplitude-dependent effects on the mean flux predicted by (3.47).

\subsection{Large-amplitude oscillations}

The computational results presented above confirm that the stability thresholds predicted by the asymptotic analysis are accurate in the limit of large membrane tension. For the smallest of the three tensions, $T=10^{3}$, the critical Reynolds number lies in a range in which our code can reliably simulate the flow. However, the growth rate is so small that it takes a long time for the oscillation to grow to a significant amplitude. To study the system's behaviour in the large-displacement regime, we therefore lowered the tension even further (to $T=10^{2}$, for which $\delta=1$ ) and investigated the system's behaviour at $R e=450$. This is well in excess of the critical Reynolds number, predicted by (3.51) to be 120.3. The steady solution for which $d^{(y)}(\zeta=5)=-0.03$ was used as the initial conditions and for $t \geqslant 0$ we set the external pressure to $p_{e}=p_{e}^{(f l u s h)}=0.96677$.

Figure 10 shows the displacement history for the central point on the membrane, computed with the same spatial discretization that we used for the small-amplitude cases. Following an initial stage during which the amplitude of the oscillation grows exponentially, the system settles into a large-amplitude limit cycle in which the wall oscillates between a strongly bulged and a strongly collapsed state. In this regime, the spatial discretization becomes too coarse to fully resolve all flow features. Therefore we performed a second simulation on a finer mesh and started the computation from a steady state in which the maximum wall displacement was close to the observed amplitude of the limit-cycle oscillation. The spatial convergence of this simulation was then confirmed by repeating it on an even finer mesh (see figure $12(\mathrm{~m})$ and figure 14 in Appendix C). Figure 10 shows that the system rapidly settles into the same limit cycle that was predicted by the computation on the coarser mesh. Thus the procedure not only allows significant savings in computational time but also demonstrates that the limit cycle is robust to fairly strong perturbations and that the system's macroscopic behaviour does not depend on the fine details of the flow field. 


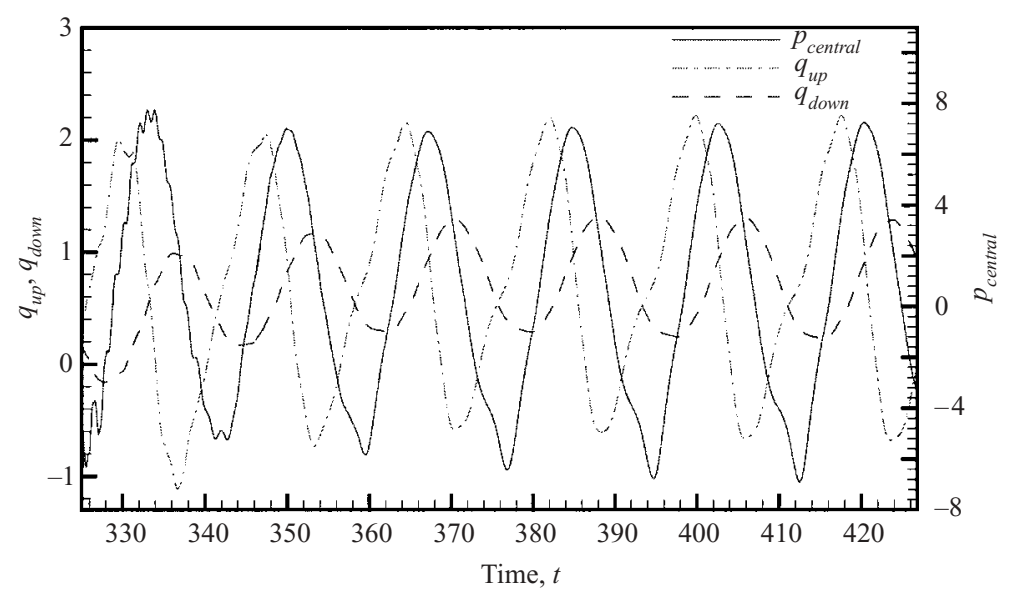

FIGURE 11. The pressure, $p_{\text {central }}$, at a nodal point in the elastic section and the fluxes $q_{u p}$ and $q_{\text {down }}$ in the upstream and downstream rigid sections, respectively. $R e=450, T=10^{2}$ and $p_{e}=p_{e}^{(f l u s h)}=0.96677$. The pressure node is located on the line $x=4.93$ and moves vertically with the deforming mesh.

Compared to the larger-tension cases considered above, the amplitude and period of the oscillation are significantly increased but qualitatively the system's behaviour is changed very little. In particular, the flow field still consists of a steady, pressuredriven flow and a superimposed high-frequency sloshing motion coupled to the wall oscillation. Figure 11 shows that the time histories of pressure and flux now contain noticeable higher-frequency components but the pressure drop and the fluxes in the upstream and downstream rigid sections are still out of phase by approximately $90^{\circ}$ (much as in figure 8), indicating that the oscillatory flow is still dominated by fluid inertia. As before, the oscillatory pressure fluctuations dominate the primary pressure drop $p_{u p}=1.2$.

Figure 12 illustrates the flow fields (pressure contours and instantaneous streamlines) during one period of the large-amplitude oscillation. In figure $12(a, b)$ the wall moves outwards but decelerates as it approaches its most strongly bulged configuration. The corresponding deceleration of the fluid causes the pressure underneath the membrane to increase strongly (although cross-stream pressure gradients remain small). The resulting adverse pressure gradient in the upstream part of the channel causes transient flow reversal inside the flexible section of the channel. The long reversed-flow region at the bottom wall then moves slowly upstream. During this phase of the oscillation, the flow generated by the outward wall motion augments the pressure-driven flux in the upstream rigid segment. Hence, the axial velocities in this part of the channel are much larger than the transverse ones, leading to an approximately uni-directional flow field. Shortly after the wall begins to move inwards (figure 12c), the wall-generated flux in the upstream rigid section becomes approximately equal and opposite to the primary, pressure-driven flux $q_{s}$ and the axial velocities become relatively small. In this situation the persisting adverse pressure gradient is large enough to create a strong flow instability which generates a complex sequence of eddies; see figure $12(d)$. As the downward wall velocity increases, the net flux in the upstream section becomes increasingly negative and the larger axial velocities cause the eddies to break up; see figure 12(e,f). Once the wall passes through the 'flush' position, the flow in the upstream section is again 

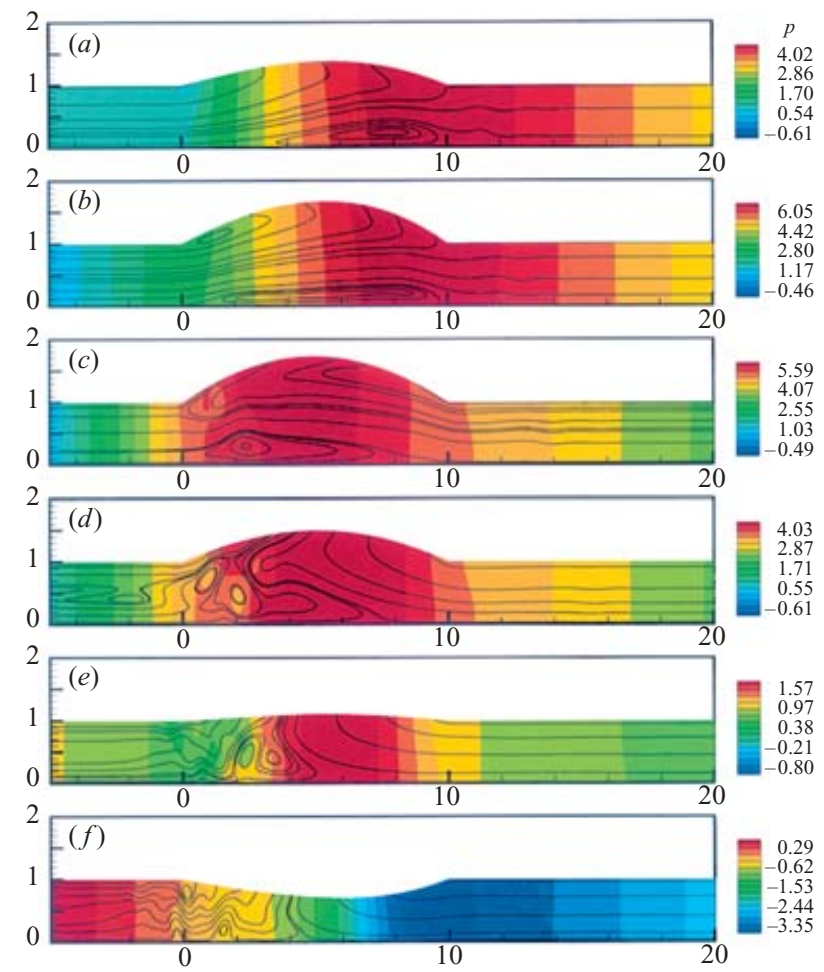
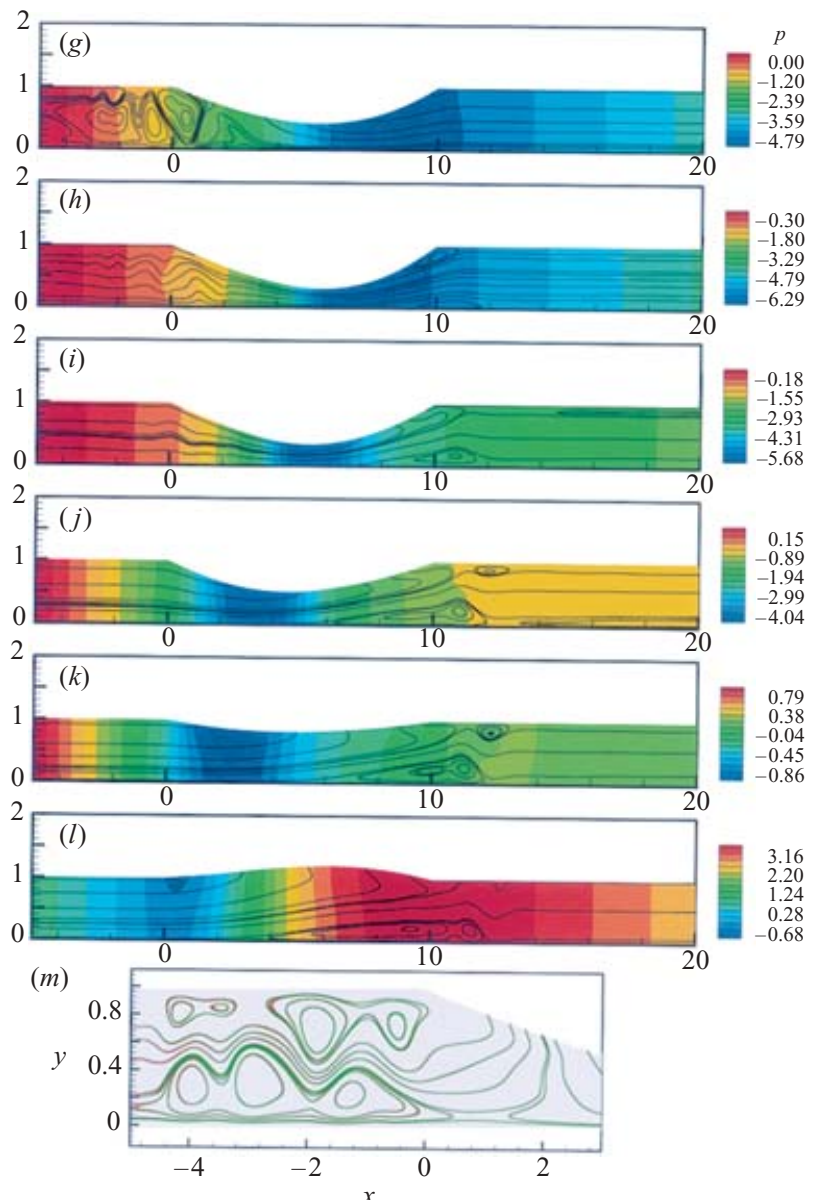

20

Figure 12. Flow fields (pressure contours and instantaneous streamlines) at equally spaced intervals of the large-amplitude oscillation. $R=450, T=10^{2}$ and $p_{e}=p_{e}^{(f l u s h)}=0.96677$. ( a $) t=347.5,(b) t=349.0$, etc. in increments of 1.5 Part $(\mathrm{m})$ presents the results of a spatial convergence test and shows the instantaneous streamlines in the upstream part of the channel at $t=340$, computed with two different spatial resolutions. Green lines: 83240 DOFs; red lines: 160140 DOFs. 
decelerated and another strong instability develops when the net flux is reduced to zero for the second time in this period; see figure $12(\mathrm{~g})$. This instability decays rapidly when the wall begins to move outwards and subjects the upstream region to a favourable pressure gradient; see figure 12( $h, i)$. During this phase of the oscillation the fluid in the downstream part of the channel is decelerated and flow reversal downstream of the collapsible segment creates a sequence of eddies on the top and bottom walls; see figure $12(j, k)$. The length of the reversed-flow region on the bottom wall increases rapidly and 'eddy doubling' can be observed before this flow structure moves upstream for the start of a new period.

While the spatial resolution of the flow fields is demonstrated by the mesh convergence study presented in figure $12(\mathrm{~m})$ and figure 14 in Appendix $\mathrm{C}$, the complexity of the flow field in the (relatively short) upstream rigid section (e.g. in figure $12 d, g$ ) raises the question of whether the boundary conditions applied at the upstream end significantly interfere with the velocity field. Ideally, one would wish to apply boundary conditions at a larger distance from the region of interest. However, in the present problem the finite length of the upstream and downstream rigid sections is an essential feature, as is the need to maintain a prescribed pressure drop over the length of the channel. The procedure employed in our computations (fixing the pressure drop by prescribing pseudo-tractions at the upstream and downstream ends) is not only the most 'forgiving' approach (see e.g. Gresho \& Sani 2000; in fact, the technique is often referred to as the 'do-nothing' boundary condition), but it also represents the natural boundary condition for the weak form of the Navier-Stokes equations. Therefore we believe that it would be difficult to do 'better'. From a practical point of view, there is no evidence of significant wave reflection from the upstream end of the channel.

\section{Discussion}

Through a combination of asymptotic analysis and numerical simulation, and by focusing on a previously unexplored region of parameter space, we are now able to present a reasonably clear mechanism for the generation of self-excited oscillations in a finite-length, deformable channel (figure 1), an idealized model of the Starling Resistor. The mechanism we have identified operates when the membrane tension is large, the Reynolds number of the mean flow driven through the channel is large and axial lengthscales are large compared to the channel width: it is probable that additional or alternative mechanisms operate in other parameter regimes.

The physical picture is as follows. In the absence of a mean flow, the finite-length channel admits a family of normal modes, governed by a balance of membrane elasticity and fluid inertia: these are global oscillations that are neutrally stable in the absence of viscous effects or a mean flow. In general, these modes are asymmetric about the mid-point of the membrane, typically because the upstream and downstream rigid channel segments are of different lengths. When the membrane tension is large, the oscillations have high frequency: in this case viscous effects are confined to oscillatory Stokes layers along both walls of the channel, which by themselves will cause the normal modes to decay. Suppose now that a flow is driven through the channel, under a fixed pressure drop, in the presence of a small-amplitude normal mode. The external agency driving the flow provides a potential source of energy. Some of this energy can be harnessed through interactions between the mean flow and the oscillation, provided there is a net imbalance in the time-averaged fluxes of 
kinetic energy at either end of the collapsible segment. The excess energy is either dissipated, or is accommodated by an increase in the amplitude of the oscillation.

This mechanism can be reinforced using a scaling argument. Suppose the primary oscillations have dimensional frequency $\omega$ and that membrane deflections are of dimensional size $D$. Other lengthscales are the channel width $a \gg D$, its length $L_{0} \gg a$ (which is also representative of the membrane length) and the width of the Stokes layers $(\mu / \rho \omega)^{1 / 2} \ll a$. Then from (2.7), pressure fluctuations have magnitude $T_{0} D / L_{0}^{2}$, and the vertical velocity has magnitude $\omega D$. Axial velocities then have size $L_{0} \omega D / a$, which generate axial pressure gradients $\rho \omega^{2} L_{0} D / a$. Balancing these with pressure gradients associated with membrane deflections $\left(T_{0} D / L_{0}^{3}\right)$ gives the frequency of oscillation to be

$$
\omega^{2} \sim \frac{T_{0} a}{\rho L_{0}^{4}},
$$

where here ' $\sim$ ' denotes 'scales like'. $(1 / \omega$ is essentially equivalent to the timescale used to scale (2.13)-(2.15).) In the presence of a mean flow with axial speed $U_{0}$, the kinetic energy flux $\rho U_{0} u^{\prime 2} a$ must balance the viscous dissipation in Stokes layers, $\mu u^{\prime 2} L_{0} /(\mu / \rho \omega)^{1 / 2}$, where $u^{\prime 2}$ denotes the mean-square axial velocity of neutrally stable oscillations. This balance yields the critical Reynolds number

$$
R e_{c} \sim\left(\frac{\rho T_{0} a}{\mu^{2}}\right)^{1 / 4}
$$

consistent with (3.51). (Since $T$ in (3.51) is scaled on $U_{0}^{2}$ via (2.8), the $T^{1 / 2}$ dependence in (3.51) translates into a $T_{0}^{1 / 4}$ dependence in (5.2).) Surprisingly, $L_{0}$ drops out of this expression, although this argument obviously fails to capture the important dependence of coefficients on the relative sizes of $L_{1}$ and $L_{2}$. The condition that the Stokes layers are thin compared to the channel width is then equivalent to $\operatorname{Re}_{c} \gg L_{0} / a$.

These insights enable us to adjust the system to promote or inhibit instability. The asymmetry in the fundamental mode is critical: when the downstream rigid segment is longer than that upstream $\left(L_{2}>L_{1}\right)$, normal modes have smaller fluxes at the downstream end of the collapsible segment than upstream, due to large downstream fluid inertia. This suppresses the kinetic energy flux out of the collapsible segment, promoting energy extraction from the mean flow at the upstream end. Oscillations are therefore promoted by increasing $L_{2}$ and reducing $L_{1}$; the limiting case of very large $L_{2}$ is equivalent instead to prescribing the flux at the channel outlet. Alternatively, no instability is predicted when $L_{1}>L_{2}$, and the extreme case of very large $L_{1}$ (prescribing the upstream flux) is the stablest possible state. The critical Reynolds number for the onset of instability is related to the membrane tension through the approximate threshold (3.51); the coefficient $r_{c 0}$ is plotted against $\hat{L}_{1}$ and $\hat{L}_{2}$ in figure 4. Given close agreement with simulations (figure 9), we can be confident of the accuracy of these predictions, but only in the parameter regime (3.50) in which the asymptotic approximation is valid, however.

The form of the resulting oscillations differs significantly from that reported previously in this system, in a number of regards. First, like Huang (2001), we find the fundamental mode has a single half-wavelength disturbance along the membrane. This is in contrast to modes reported in other studies (both one-dimensional, e.g. Jensen 1990, 1992 and two-dimensional, e.g. Luo \& Pedley 1996), where, because the membrane tension is lower, the basic state is strongly influenced by the viscous pressure drop along the channel, so that typically the membrane is inflated at its upstream end 
and collapsed downstream, a shape which appears to favour disturbances having two half-wavelengths along the membrane (Jensen 1990, 1992). Second, our simulations show only limited evidence of vorticity waves (large-amplitude Tollmien-Schlichting waves) propagating into the downstream rigid segment (although there is a suggestion of such waves being present in figure $12 a-c$ ). These were a major feature of previous simulations (Luo \& Pedley 1996), but do not appear with any strength here because with large membrane tension the resulting Strouhal number is large enough to fall into a regime in which Tollmien-Schlichting waves do not appear to be excited (Rosenfeld 1995). Instead, we find a rich variety of different secondary instabilities, which are particularly evident upstream of the membrane, when the upstream-directed flux due to the oscillation has a magnitude close to that of the imposed mean flow. At large amplitudes, vigorous wall motions induce considerable transverse velocities in the flow field beneath the elastic segment, in contrast to Luo \& Pedley's simulations where, apart from small regions near the point of separation, the instantaneous streamlines were practically tangential to the moving wall. This relates to a third major difference with previous studies, concerning flow separation: steady separation beyond the point of strongest collapse was previously identified as a major factor controlling the origin of instabilities (Cancelli \& Pedley 1985). This viewpoint was questioned first when Hayashi, Hazase \& Kawamura (1998) reproduced self-excited oscillations in a one-dimensional model using a distributed friction term, and later when Luo \& Pedley (1996) found that the dominant dissipation in steady flow occurred in viscous boundary layers upstream of the constriction, and not in the region of separated flow. The present study goes further, showing that small-amplitude oscillations can spontaneously grow in magnitude in the absence of separation. Nevertheless, onedimensional models (Cancelli \& Pedley 1985; Jensen 1992) do still suggest that the delicate physics controlling unsteady separation may have a profound effect on the origin of instabilities elsewhere in parameter space. Finally, to emphasize the richness of this problem, we note that Luo \& Pedley (1996) observed self-excited oscillations when the upstream flux was held constant, a regime which the present model predicts to be strongly stable. (While their results show a trend of $R e_{c}$ increasing with $T_{0}$, their limited data do not convincingly exhibit the 1/4-power relationship in (5.2).) Evidently effects which are not relevant for the instability analysed in the present study (such as the non-uniformity of the basic state, flow separation or possibly vorticity waves) may play a major role in alternative instability mechanisms.

Finally, we have found that asymptotics and computation have proved a powerful combination with which to investigate this problem. Computations have validated the accuracy of the approximations, which work respectably even when the primary small parameter $\delta$ was as large as 0.3 (figure 9), and the picture that was established for $\delta \ll 1$ proved useful in interpreting results obtained for the case $\delta=1$ (figure 12). Likewise, insights from the asymptotics were invaluable in guiding expensive computations towards dynamically interesting regions of parameter space. The asymptotics also revealed the complex manner in which effects such as membrane distortion or steady streaming can influence the energy transfer between the mean flow and oscillations, which this study has only begun to unravel. The challenge now is to extend this combined approach into regimes more relevant to the Starling Resistor and its physiological applications, and to see to what extent the mechanism of instability identified here applies to these more general situations.

Financial support from the EPSRC for an Advanced Fellowship for M.H. is gratefully acknowledged. M.H. also wishes to thank Dr Andrew Hazel for many 
helpful and enjoyable discussions. The HSL library routine MA42 (a frontal solver for sparse, unsymmetric systems) was used in our code.

\section{Appendix A. Steady streaming}

To find $\boldsymbol{u}_{10}$, which satisfies $\nabla \cdot \boldsymbol{u}_{10}=0, u_{10} \sim|A|^{2} u_{s}$ as $y \rightarrow 0$ and $u_{10} \sim|A|^{2} u_{s}+$ $6 q_{00} h_{00}$ as $y \rightarrow 1$ (see $\left.(3.26 a, b)\right)$, we consider the equation governing $\omega_{10}=-u_{10 y}$ (3.43), which we write as

$$
\omega_{10 t_{1}}+\left(\boldsymbol{u}_{00} \cdot \nabla\right) \omega_{10}+\left(\boldsymbol{u}_{10} \cdot \nabla\right) \omega_{00}=\delta \omega_{10 y y} / r^{2} .
$$

In terms of the streamfunction $\psi_{10}$ (for which $u_{10}=\psi_{10 y}$ ), and assuming $\omega_{10 t_{1}}=0$, (A 1) becomes

$$
y(1-y) \psi_{10 x y y}+2 \psi_{10 x}=\varepsilon^{3} \psi_{10 y y y y}, \quad \varepsilon \equiv\left(\delta / 6 q_{00} r\right)^{1 / 3} .
$$

Here $\varepsilon^{-3}$ is equivalent to a steady-streaming Reynolds number. We examine the solution of (A 2) for $\varepsilon \ll 1$.

In the core of the channel $y(1-y) \psi_{10 y y}+2 v_{10}=0$ at leading order (a Rayleigh equation with critical layers at each wall), implying

$$
v_{10}=-\psi_{10 x}=-B_{x} y(1-y)-q_{10 x}\left[y(1-y) \log \left(\frac{y}{1-y}\right)-\frac{1}{2}+y\right]
$$

for some functions $B(x), q_{10}(x)$, where $q_{10}=\int_{0}^{1} u_{10} \mathrm{~d} y$ is a flux. It follows that

$$
\begin{array}{ll}
u_{10} \sim B+q_{10}[\log y+2] & (y \rightarrow 0), \\
u_{10} \sim-B+q_{10}[\log (1-y)+2] & (y \rightarrow 1) .
\end{array}
$$

The $B$ contribution to $u_{10}$ is antisymmetric about $y=\frac{1}{2}$, and the $q_{10}$ term is symmetric. We therefore rewrite the boundary conditions on $u_{01}$ as

$$
\begin{array}{ll}
u_{10} \sim\left[|A|^{2} u_{s}+3 q_{00} h_{00}\right]-3 q_{00} h_{00} & (y \rightarrow 0), \\
u_{10} \sim\left[|A|^{2} u_{s}+3 q_{00} h_{00}\right]+3 q_{00} h_{00} & (y \rightarrow 1) .
\end{array}
$$

Thus at leading order in $\varepsilon$,

$$
B=-3 q_{00} h_{00}=\frac{3}{2} \tilde{p}_{e} q_{00} x(1-x)
$$

and $q_{10}=o(1)$, yielding a single vortex in $\boldsymbol{u}_{10}$ that rotates anticlockwise when $\tilde{p}_{e}>0$. In conjunction with the mean flow $\boldsymbol{u}_{00}$, this corresponds to the sideways displacement of streamlines in response to indentation of the membrane.

The symmetric component of the flow is controlled by viscous boundary layers on each wall. Writing

$$
y=\varepsilon Y, \quad \psi_{10}=-3 q_{00} h_{00} y+\varepsilon \Psi_{10}(x, Y),
$$

or

$$
y=1-\varepsilon Y, \quad \psi_{10}=-3 q_{00} h_{00}(1-y)-\varepsilon \Psi_{10}(x, Y)
$$

on the lower and upper walls respectively, we have in each boundary layer

$$
\begin{array}{r}
Y \Psi_{10 x Y Y}+\varepsilon\left[2 \Psi_{10 x}-Y^{2} \Psi_{10 x Y Y}\right]=\Psi_{10 Y Y Y Y}, \\
\Psi_{10}(x, 0)=0, \quad \Psi_{10 Y}(x, 0)=|A|^{2} u_{s}(x)+3 q_{00} h_{00} .
\end{array}
$$

Here $Y$ is distinct from the inner variable in the Stokes layers. The streaming induced in each boundary layer by the boundary condition on $\Psi_{10 Y}$ induces a vertical flow of 

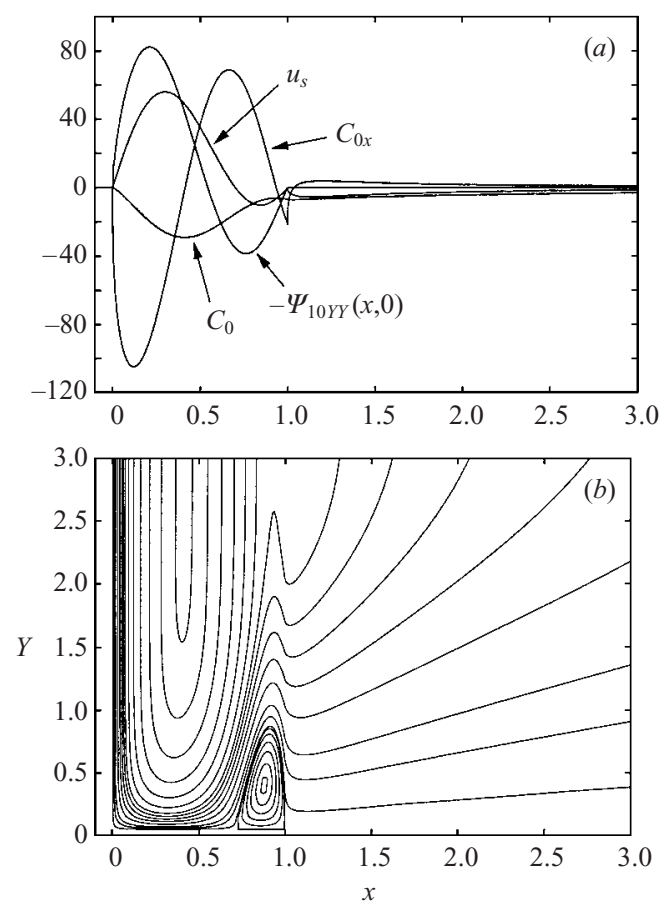

FiguRE 13. Flow in the steady-streaming boundary layer, driven by $u_{s}(x)$ with $\tilde{p}_{e}=0$, for $\hat{L}_{1}=0.5, \hat{L}_{2}=3,|A|=1$. (a) $u_{s}(x),-\Psi_{10 Y Y}$ along $Y=0, C_{0}(x)$ (displacement thickness) and $C_{0 x}$ (vertical velocity as $Y \rightarrow \infty$ ). (b) Contours of $\Psi_{10}$; contour values were chosen to illustrate flow structures and are non-uniformly spaced.

$O(\varepsilon)$ at the boundary-layer outer edge, forcing a recirculation in the core through the leading-order outer boundary condition

$$
\Psi_{10} \sim-C_{0}(x) \text { as } Y \rightarrow \infty, \text { where } q_{10}=2 \varepsilon C_{0}(x) .
$$

The symmetric recirculation in the core (represented by the $q_{10 x}$ term in (A 3)) then induces a horizontal flow of $O(\varepsilon \log \varepsilon)$ at the outer edge of each boundary layer (see (A4)), which reduces to zero across the boundary layer in order to satisfy no slip. There is a further $O(\varepsilon)$ correction where $O(1)$ vorticity at the boundary-layer outer edge arises through critical-layer effects, and thereafter a sequence of higher-order corrections. The corresponding contributions to the flux are $O\left(\varepsilon^{2} \log \varepsilon\right)$ and $O\left(\varepsilon^{2}\right)$, but we do not compute them here.

To illustrate this flow, we show the leading-order boundary layer flow satisfying (A 8 ) in the case $\hat{L}_{1}=0.5, \hat{L}_{2}=3, \tilde{p}_{e}=0,|A|=1$ and $\epsilon \rightarrow 0$. This was computed using a finite-difference method based on the Crank-Nicholson scheme, marching forward in $x$ and giving second-order accuracy in both spatial directions. Figure 13(a) shows the driving $u_{s}$ distribution, which is forwards in the upstream two-thirds and reversed in the downstream third of the membrane, and the resulting vertical flow $C_{0 x}(x)$ induced outside the boundary layer. Figure $13(b)$ shows streamlines in the boundary layer. Fluid is drawn downwards strongly near $x=0$ and pushed upwards further downstream, with a small closed eddy forming near $x=1$. This flow drives a weak recirculation in the core above, which extends beyond $x=1$. 
Careful inspection of numerical simulations of the full unsteady flow failed to reveal the $O\left(\delta^{1 / 3}|A|^{2}\right)$ recirculation in the core driven by steady streaming alone. This is because it is typically dominated by the stronger sideways displacement flow of $O\left(\tilde{p}_{e}\right)$.

\section{Appendix B. Energetics of neutrally stable oscillations}

Here we examine the energy equation (2.15) in the special case of a perfectly periodic, small-amplitude oscillation. First we set $\boldsymbol{u}=\overline{\boldsymbol{u}}+\boldsymbol{u}^{\prime}$, where $\overline{\boldsymbol{u}}$ is steady and $\boldsymbol{u}^{\prime}$ is periodic with zero mean, with $\left|\boldsymbol{u}^{\prime}\right| \ll 1$. We substitute into (2.15) and take the time average over one oscillation, denoting the time average with an overbar. This procedure is followed for $-\hat{L}_{1}<x<0,0<x<1$ and $1<x<\hat{L}_{2}$ separately, and the energy equations for each region are added.

The term representing rate of change of kinetic energy vanishes after taking a time average. The energy fluxes, and the terms representing the rate at which pressure does work on the axial flow, when added, yield the difference between fluxes at $x=-\hat{L}_{1}$ and $x=1+\hat{L}_{2}$,

$$
\left[\int_{0}^{1}\left(\frac{\delta}{2}\left(\bar{u}^{3}+3 \bar{u} \overline{u^{\prime 2}}\right)+\bar{u} \bar{p}+\overline{u^{\prime} p^{\prime}}\right) \mathrm{d} y\right]_{-\hat{L}_{1}}^{1+\hat{L}_{2}} .
$$

Cubic terms in primed quantities have here been neglected. Pressure forces do zero work on the membrane over one oscillation, since (from $(2.12 d)$ ) we can write

$$
\left.\int_{0}^{1}(p v)\right|_{y=h} \mathrm{~d} x=\int_{0}^{1}\left(\tilde{p}_{e}-h_{x x}\right) h_{t} \mathrm{~d} x=\left[\int_{0}^{1}\left(\tilde{p}_{e} h+\frac{1}{2} h_{x}^{2}\right) \mathrm{d} x\right]_{t} .
$$

The dissipation in the flow is dominated by that in the Stokes layers, since

$$
\frac{\delta^{2}}{r^{2}} \int_{0}^{h} \overline{u_{y}^{2}} \mathrm{~d} y=2 \frac{\delta}{r} \int_{0}^{\infty} \overline{U_{Y}^{2}} \mathrm{~d} Y+\frac{\delta^{2}}{r^{2}} \int_{0}^{1} \overline{u_{y}^{2}} \mathrm{~d} y
$$

where the first integral is over one of the Stokes layers (both have the same structure to leading order) and the second over the core.

Taking

$$
\overline{\boldsymbol{u}}=\boldsymbol{u}_{00}+\delta \boldsymbol{u}_{10}+O\left(\delta^{2}\right), \quad \boldsymbol{u}^{\prime}=\boldsymbol{u}_{01} \mathrm{e}^{\mathrm{i} \beta t}+\overline{\mathbf{u}}_{01} \mathrm{e}^{-\mathrm{i} \beta t}+O(\delta),
$$

and exploiting what we know from $\S \S 3.1-3.5$ about $\boldsymbol{u}_{00}, \boldsymbol{u}_{01}$ and $\boldsymbol{u}_{10}$, (B 1)-(B 3) together give at leading order

$$
\begin{aligned}
& \frac{3 \delta}{2}\left[\int_{0}^{1} u_{00}^{2} u_{10} \mathrm{~d} y\right]_{0}^{1+\hat{L}_{2}}+3 \delta q_{00}\left[u_{01} \bar{u}_{01}\right]_{0}^{1}-\frac{12\left(1+\hat{L}_{1}+\hat{L}_{2}\right)}{r^{2}} \delta^{2} q_{00} \\
& \quad+\left[u_{01} \bar{p}_{01}+\bar{u}_{01} p_{01}+O\left(\delta|A|^{3}\right)\right]_{0}^{1} \\
& \quad+\frac{4 \delta}{r} \int_{-\hat{L}_{1}}^{1+\hat{L}_{2}} \int_{0}^{\infty}\left(U_{01 Y} \bar{U}_{01 Y}+\ldots\right) \mathrm{d} Y \mathrm{~d} x+\frac{\delta^{2}}{r^{2}} \int_{-\hat{L}_{1}}^{1+\hat{L}_{2}} \int_{0}^{1} u_{00 y}^{2} \mathrm{~d} y \mathrm{~d} x=0 .
\end{aligned}
$$

As indicated in $\$ 3.3 .1$, with pressure-driven flow we expect $|A|=o(1)$. As described in $\S 3.5$ and Appendix A, $u_{10}=O\left(\delta^{1 / 3}|A|^{2}\right)+O\left(\delta^{1 / 3} \tilde{p}_{e}\right)$ in the core at $x=0$ and $x=1$. Thus the kinetic energy flux due to steady streaming is subdominant by $\delta^{1 / 3}$ to that due to the primary oscillation; we may also neglect the net energy flux due to membrane indentation, since the component of $u_{10}$ due to $\tilde{p}_{e}$ vanishes at $x=0$ and decays along the downstream rigid segment. Also $u_{01} \bar{p}_{01}+\bar{u}_{01} p_{01}=0$ (from (3.14)), 


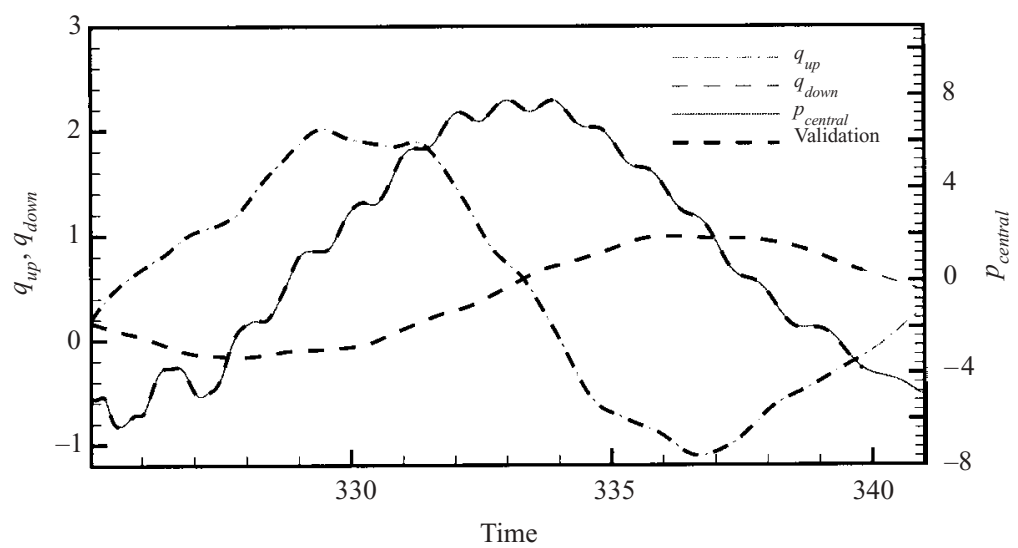

Figure 14. Convergence test for the large-amplitude oscillation: Fluxes in the upstream and downstream rigid segments and the pressure at a central node. Thin lines: 83240 DOFs; thick dashed line: 160140 DOFs. $R e=450, T=10^{2}$ and $p_{e}=p_{e}^{(f l u s h)}=0.96677$. The pressure node is located on the line $x=4.93$ and moves vertically with the deforming mesh.

and the next correction to work done by pressure on the fluid at $O\left(\delta|A|^{3}\right)$ may also be neglected. Provided $\tilde{p}_{e}=o\left(\delta^{2 / 3}\right)$, we may therefore write the energy budget (B 5) as $\mathscr{F}+\mathscr{P}=\mathscr{D}_{S}+\mathscr{D}_{P}$, where

$$
\begin{gathered}
\mathscr{F}=-\frac{3 \delta}{\beta^{2}} q_{00}|A|^{2}\left[\phi_{01 x x x}^{2}\right]_{0}^{1}, \\
\mathscr{P}=12\left(1+\hat{L}_{1}+\hat{L}_{2}\right) \delta^{2} q_{00} / r^{2}, \\
\mathscr{D}_{S}=\frac{4 \delta}{r} \frac{|A|^{2}}{2^{1 / 2} \beta^{3 / 2}}\left[\int_{0}^{1} \phi_{01 x x x}^{2} \mathrm{~d} x+\hat{L}_{1} \phi_{01 x x x}^{2}(0)+\hat{L}_{2} \phi_{01 x x x}^{2}(1)\right], \\
\mathscr{D}_{P}=12\left(1+\hat{L}_{1}+\hat{L}_{2}\right) \delta^{2} q_{00}^{2} / r^{2} .
\end{gathered}
$$

We may use (3.16) to re-write the net flux of kinetic energy into the system $(\mathscr{F})$ and the dissipation in the Stokes layers $\left(\mathscr{D}_{S}\right)$ as

$$
\mathscr{F}=3 \delta q_{00}|A|^{2}\left[\phi_{01 x}^{2}\right]_{0}^{1}, \quad \mathscr{D}_{S}=\frac{2 \delta}{r}|A|^{2}(2 \beta)^{1 / 2} \int_{0}^{1} \phi_{01 x}^{2} \mathrm{~d} x .
$$

Furthermore, since $r=r_{c}$ when oscillations are neutrally stable, we can use (3.38) to deduce $\mathscr{D}_{S}=2 \mathscr{F} / 3$, or equivalently that

$$
\frac{r}{r_{c}}=\frac{2 \mathscr{F}}{3 \mathscr{D}_{S}} .
$$

Instability $\left(r>r_{c}\right)$ then has a clear interpretation as the rate at which kinetic energy is extracted from the mean flow exceeding three-halves the rate of viscous energy dissipation in the Stokes layers. With $r=r_{c}$, the energy equation then reduces to

$$
q_{00}=1+\frac{|A|^{2}}{\delta} \frac{r_{c}^{2}\left[\phi_{01 x}^{2}\right]_{0}^{1}}{12\left(1+\hat{L}_{1}+\hat{L}_{2}\right)}=1+\frac{\mathscr{F} / 3}{\mathscr{D}_{P} / q_{00}}
$$

(or equivalently $\mathscr{D}_{P}=\mathscr{P}+(\mathscr{F} / 3)$ ), which is in agreement with the flux-amplitude relation obtained directly in (3.47). We can interpret this result as follows: the energy source driving the oscillation is the flux $\mathscr{F}$ of kinetic energy extracted from the mean 
flow: for a neutrally stable oscillation, two-thirds of this energy is lost to dissipation in Stokes layers $\left(\mathscr{D}_{S}=2 \mathscr{F} / 3\right)$, and the remaining third must be balanced by increased viscous dissipation by the mean flow (B9). Thus neutrally stable small-amplitude oscillations cause the mean flux to increase.

\section{Appendix C. Convergence tests for large-amplitude simulations}

Figures $12(\mathrm{~m})$ and 14 present results of the spatial convergence tests for the largeamplitude oscillation discussed in $\S 4.2$. Figure 14 compares the predictions for the upstream and downstream fluxes and the pressure at a central point the elastic section, obtained with two different spatial discretizations. Figure $12(\mathrm{~m})$ compares the instantaneous streamlines at $t=340$, an instant at which the flow in the upstream rigid section is at its most complex.

\section{REFERENCES}

Bertram, C. D. \& Pedley, T. J. 1982 A mathematical model of unsteady collapsible tube behaviour. J. Biomech. 15, 39-50.

Bertram, C. D., Raymond, C. J. \& Pedley, T. J. 1990 Mapping of instabilities for flow through collapsible tubes of differing length. J. Fluids Struct. 4, 125-153.

Bertram, C. D., Raymond, C. J. \& Pedley, T. J. 1991 Application of nonlinear dynamics concepts to the analysis of self-excited oscillations of a collapsible tube conveying a fluid. J. Fluids. Struct. 5, 391-426.

Bogdanova, E. V. \& Ryzhov, O. S. 1983 Free and induced oscillations in Poiseuille flow. Q. J. Mech. Appl. Maths. 36, 271-287.

Bogner, F. K., Fox, R. L. \& Schmit, L. A. 1967 A cylindrical shell discrete element. AIAA J. 5, 645-750.

Cancelli, C. \& Pedley, T. J. 1985 A separated-flow model for collapsible-tube oscillations. J. Fluid Mech. 157, 375-404.

Davies, C. \& CARpenter, P. W. 1997 Instabilities in a plane channel flow between compliant walls. J. Fluid Mech. 352, 205-243.

Duck, P. W. 1979 Viscous flow through unsteady symmetric channels. J. Fluid Mech. 95, 635-653.

GaJJar, J. S. B. \& Sibanda, P. 1996 The hydrodynamic stability of channel flow with compliant boundaries. Theor. Comput Fluid Dyn. 8, 105-131.

Gresho, P. M. \& SANI, R. L. 2000 Incompressible Flow and the Finite Element Method. Chichester: Wiley.

Grotberg, J. B. \& Reiss, E. L. 1984 Subsonic flapping flutter. J. Sound Vib. 92, 349-361.

Guneratne, J. C. 1999 High-Reynolds number flow in a collapsible channel. PhD Thesis, University of Cambridge.

Hayashi, S., Hazase, T. \& Kawamura, H. 1998 Numerical analysis for stability and self-excited oscillation in collapsible tube flow. J. Biomech. Engng 120, 468-475.

Hazel, A. L. \& Heil, M. 2003 Steady finite-Reynolds-number flows in three-dimensional collapsible tubes. J. Fluid Mech. (in press).

HeIL, M. 1997 Stokes flow in collapsible tubes: computation and experiment. J. Fluid Mech. 353, 285-312.

HeIL, M. 1998 Stokes flow in an elastic tube - a large-displacement fluid-structure interaction problem. Intl J. Numer. Meth. Fluids 28, 243-265.

HeIL, M. 2000 Finite Reynolds number effects in the propagation of an air finger into a liquid-filled flexible-walled channel. J. Fluid Mech. 424, 21-44.

Heil, M. \& Jensen, O. E. 2003 Flows in deformable tubes and channels - theoretical models and biological applications. In Flow in Collapsible Tubes and Past Other Highly Compliant Boundaries (ed. T. J. Pedley \& P. W. Carpenter). Academic.

Heil, M. \& White, J. P. 2002 Airway closure: Surface-tension-driven non-axisymmetric instabilities of liquid-lined elastic rings. J. Fluid Mech. 462, 79-109. 
HuAng, L. 2001 Viscous flutter of a finite elastic membrane in Poiseuille flow. J. Fluids Struct. 15, $1060-1088$.

Jensen, O. E. 1990 Instabilities of flow in a collapsed tube. J. Fluid Mech. 220, 623-659.

JENSEN, O. E. 1992 Chaotic oscillations in a simple collapsible-tube model. J. Biomech. Engng 114, $55-59$.

Kamm, R. D. \& Pedley, T. J. 1989 Flow in collapsible tubes: a brief review. J. Biomech. Engng 111, $177-179$.

Kistler, S. F. \& SCRIVEn, L. E. 1983 Coating flows. In Computational Analysis of Polymer Processing (ed. J. Pearson \& S. Richardson). Applied Science Publishers.

Luo, X. Y. \& Pedley, T. J. 1995 Numerical simulation of steady flow in a 2-D collapsible channel. J. Fluids Struct. 9, 149-197.

Luo, X. Y. \& Pedley, T. J. 1996 A numerical simulation of unsteady flow in a two-dimensional collapsible channel. J. Fluid Mech. 314, 191-225.

Luo, X. Y. \& Pedley, T. J. 1998 The effects of wall inertia on flow in a two-dimensional collapsible channel. J. Fluid Mech. 363, 253-280.

Luo, X. Y. \& Pedley, T. J. 2000 Multiple solutions and flow limitation in collapsible channel flows. J. Fluid Mech. 420, 301-324.

Pedley, T. J. \& LuO, X. Y. 1998 Modelling flow and oscillations in collapsible tubes. Theor. Comput. Fluid Dyn. 10, 277-294.

Pedley, T. J. \& Stephanoff, K. D. 1985 Flow along a channel with a time-dependent indentation in one wall: the generation of vorticity waves. J. Fluid Mech. 160, 337-367.

Ralph, M. E. \& Pedley, T. J. 1988 Flow in a channel with a moving indentation. J. Fluid Mech. 190, 87-112.

Ralph, M. E. \& Pedley, T. J. 1989 Viscous and inviscid flow in a channel with a moving indentation. J. Fluid Mech. 209, 543-566.

Ralph, M. E. \& Pedley, T. J. 1990 Flow in a channel with a moving indentation in one wall. Trans. ASME: J. Fluids Engng 112, 468-475.

RAST, M. P. 1994 Simultaneous solution of the Navier-Stokes and elastic membrane equations by a finite-element method. Intl J. Numer. Meth. Fluids 19, 1115-1135.

Riley, N. 2001 Steady streaming. Annu. Rev. Fluid Mech. 33, 43-65.

Rosenfeld, M. 1995 A numerical study of pulsating flow behind a constriction. J. Fluid Mech. 301, 203-223.

Sani, R. L., Gresho, P. M., Lee, R. L. \& Griffith, D. F. 1981a The cause and cure of the spurious pressures generated by certain FEM solutions of the incompressible Navier-Stokes equations: Part 1. Intl J. Numer. Meth. Fluids 1, 17-43.

Sani, R. L., Gresho, P. M., Lee, R. L. \& Griffith, D. F. $1981 b$ The cause and cure of the spurious pressures generated by certain FEM solutions of the incompressible Navier-Stokes equations: Part 2. Intl J. Numer. Meth. Fluids 1, 171-204.

Secomb, T. W. 1979 Flows in channels and tubes with indented and moving walls. PhD Thesis, University of Cambridge.

Shapiro, A. H. 1977 Steady flow in collapsible tubes. J. Biomech. Engng 99, 126-147.

Sмith, F. T. $1976 a$ Flow through constricted or dilated pipes and channels. Part 1. Q. J. Mech. Appl. Maths 21, 343-364.

Sмith, F. T. $1976 b$ Flow through constricted or dilated pipes and channels. Part 2. Q. J. Mech. Appl. Maths 21, 365-379.

Sobey, I. J. 1985 Observation of waves during oscillatory channel flow. J. Fluid Mech. 151, 395-426.

TutTy, O. R. 1992 Pulsatile flow in a constricted channel. J. Biomech. Engng 114, 50-54.

Tutty, O. R. \& Pedley, T. J. 1993 Oscillatory flow in a stepped channel. J. Fluid Mech., 247, 179-204. 